\title{
Exploiting the Site Selectivity of Perfluoropyridine for Facile Access to Densified Polyarylene Networks for Carbon-Rich Materials
}

\author{
Matthew B. Houck ${ }^{\dagger}$, Loren C. Brown ${ }^{\dagger}$, Robert H. Lambeth ${ }^{\ddagger}$, and Scott T. Iacono ${ }^{+, *}$ \\ ${ }^{*}$ Correspondence e-mail: scott.iacono@usafa.edu \\ 'Department of Chemistry and Chemistry Research Center, \\ Laboratories for Advanced Materials, United States Air Force Academy, \\ Colorado Springs, Colorado (USA) \\ ${ }^{\ddagger}$ Composites and Hybrid Materials Branch, Army Research Laboratory \\ Aberdeen Proving Grounds, Maryland (USA)
}

\section{Table of Contents}

1. Methods and materials

2. Experimental procedures and characterization data

3. NMR spectra

4. Thermal Analysis

5. X-Ray Data

6. References
S2

S3

S11

S29

S32

S34 


\section{Methods and materials}

All the key reactions were carried out under nitrogen atmosphere with a stir bar in flame-dried, sealed round bottom unless otherwise noted. Pentafluoropyridine was obtained from SynQuest Laboratories, Naphthalen-2-ol and 9-bromoanthracene were obtained from Oakwood Chemical. 1-Bromo-4-(phenylethynyl)benzene was obtained from TCl Chemical. tert-Butyl lithium (1.7 M in pentane) was obtained from Sigma. All other materials were obtained from commercial sources and were used as received unless otherwise specified.

Unless otherwise stated, ${ }^{1} \mathrm{H}$ NMR ${ }^{19} \mathrm{~F}$ NMR, and ${ }^{13} \mathrm{C}$ NMR spectra were recorded on a Jeol (500 MHz) spectrometer. Chemical shifts were reported in parts per million (ppm), and the residual solvent peak was used as an internal reference: proton (chloroform $\delta$ 7.26), carbon (chloroform $\delta 77.0)$, fluorine $\left(\mathrm{CFCl}_{3} \delta 0.00\right)$ or tetramethylsilane (TMS $\left.\delta 0.00\right)$ was used as a reference. Data are reported as follows: chemical shift, multiplicity $(s=$ singlet, $d=$ doublet, $t=$ triplet, $\mathrm{dd}=$ doublet of doublets, $\mathrm{td}=$ triplet of doublets, $\mathrm{dt}=$ doublet of triplets, $\mathrm{ddd}=$ doublet of doublet of doublets, $m=$ multiplet, $b s=$ broad singlet, etc.), coupling constants $(\mathrm{Hz})$ and integration.

High resolution mass spectra (HRMS) were obtained on a Synapt G2 HDMS instrument using positive mode. Flash chromatography was carried out on a YAMAZEN Smart Flash EPCLC W-Prep $2 \mathrm{XY}$ system with a $10 \mathrm{~g}$ Biotage SNAP KP-Sil Cartridge silica gel column as the stationary phase. Melting points and monomer curing were performed by differential scanning calorimetry using a TA DSC Q20 DSC utilizing aluminum hermetic pans and a heating rate of $5{ }^{\circ} \mathrm{C} / \mathrm{s}$. All experiments were performed under nitrogen. The thermal stability and char behavior of the thermosets was determined by thermogravimetric analysis using a TA TGA Q500 utilizing 
platinum pans and a heating rate of $5^{\circ} \mathrm{C} / \mathrm{s}$. All experiments were performed under argon unless otherwise noted.

Gas chromatography mass spectrometry (GC/MS) analyses were performed on an Agilent 7890 gas chromatography coupled to an Agilent 5975C electron impact mass spectrometer with a temperature gradient of $80^{\circ} \mathrm{C}-250^{\circ} \mathrm{C}$ at $15^{\circ} \mathrm{C} / \mathrm{min}$ after an initial $2 \mathrm{~min}$ temperature hold at $80^{\circ} \mathrm{C}$.

Single crystal X-ray diffraction data was collected using a Rigaku XtaLAB Synergy instrument consisting of a photonJet Mo source and a HYPIX dectector. Crystals were centered on the goniometer of a Rigaku Synergy S diffractometer operating with MoK radiation. The data collection routine, unit cell refinement, and data processing were carried out with the program CrysAlisPro. The Laue symmetry and systematic absences were consistent with the triclinic space group P-1. The structure was solved using SHELXT and refined using SHELXL via Olex2. The final refinement model involved anisotropic displacement parameters for non-hydrogen atoms and a riding model for all hydrogen atoms. Olex2 and Mercury were used for molecular graphics generation.

\section{Experimental procedures}

\subsection{General procedure for the lithiation of aryl bromides}

t-BuLi (1.18 $\mathrm{mL}, 2$ molar equiv., $1.7 \mathrm{M}$ in pentane) was added dropwise to the selected aryl bromide (vida infra) dissolved in diethyl ether $(30 \mathrm{~mL})$ at $-78^{\circ} \mathrm{C}$. The reaction mixture was stirred for $1 \mathrm{~h}$ at $-78^{\circ} \mathrm{C}$ at which point lithiation was complete as confirmed by GC-MS analysis from quenched aliquots. The resulting lithiated species was then used in situ for the preparation of $\mathbf{1}$,

$1 a, 1 b, 2,2 a$, and $2 b$. 


\subsection{Synthesis of 2,3,5,6-tetrafluoro-4-(4-(phenylethynyl)phenyl)pyridine (1)}

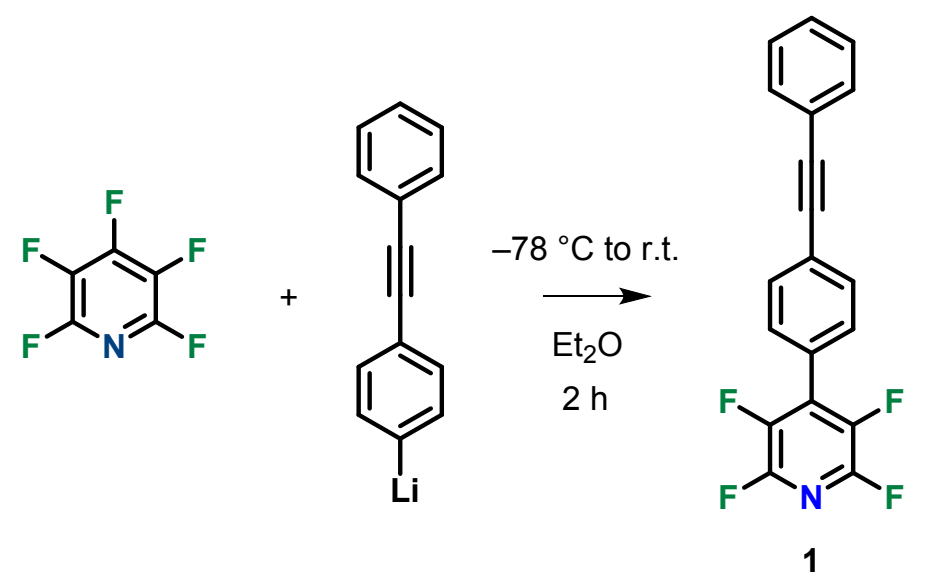

Lithiated diphenylacetylene (generated from $1.521 \mathrm{~g}, 5.915$ mmol 1-bromo-4(phenylethynyl)benzene) in diethyl ether $(30 \mathrm{~mL}, 0.1972 \mathrm{M})$ at $-78{ }^{\circ} \mathrm{C}$ was added dropwise Perfluoropyridine $(1.008 \mathrm{~g}, 5.963 \mathrm{mmol})$ dissolved in diethyl ether $(15 \mathrm{~mL})$ at $-78^{\circ} \mathrm{C}$ over a period of 10 min, during this time liquid $\mathrm{N}_{2}$ was added periodically to maintain the low temperature of the cold bath. Upon initial addition the reaction mixture turned a deep red/purple and the reaction mixture was allowed to warm to room temperature during which time the reaction mixture turned bright yellow. After $2 \mathrm{~h}$, the reaction was quenched by dropwise addition of methanol $(1 \mathrm{~mL})$, followed by the addition of saturated, aqueous ammonium chloride. The top ether layer was extracted $3 x$, dried over magnesium sulfate, and then concentrated under reduced pressure to give a red viscous oil. The crude product was passed through a silica gel flash column using hexanes/ethyl acetate $\left(R_{f} 0.81,75: 25, v / v\right)$ as the eluent. The collected fractions were vacuum dried to give the desired product as a fluffy, white solid (1.469 g, 75\%). ${ }^{1} \mathrm{H}$ NMR (500 MHz, chloroform- $d$ ) $\delta 7.69(\mathrm{~d}, J=8.5 \mathrm{~Hz}, 2 \mathrm{H}), 7.56$ (dd, J=3.5, 2.7 Hz, 2H), $7.54(\mathrm{~d}, J=8.5 \mathrm{~Hz}$, $2 \mathrm{H}), 7.38(\mathrm{dt}, J=3.5,2.7 \mathrm{~Hz}, 3 \mathrm{H})(9 \mathrm{H}) ;{ }^{19} \mathrm{~F}$ NMR (471 MHz, chloroform-d) $\delta-87.62-(-92.65)(\mathrm{m}$, 2F), -144.21-(-146.32) (m, 2F); ${ }^{13} \mathrm{C}$ NMR (126 MHz, chloroform-d) $\delta 145.32$ - 144.89 (td, $\left.16.3 \mathrm{~Hz}\right)$, 
$143.16(\mathrm{td}, J=16.1 \mathrm{~Hz}), 140.80-139.90(\mathrm{dm}, 34.2 \mathrm{~Hz}), 138.78-137.74(\mathrm{dm}, 35.1 \mathrm{~Hz}), 132.05$,

$131.83,128.88,128.54,125.98,125.48,122.74,91.89,88.40 ;$ HRMS (ESI) calcd. for $\mathrm{C}_{19} \mathrm{H}_{10} \mathrm{~F}_{4} \mathrm{~N}$ $[\mathrm{M}+\mathrm{H}]^{+}: 328.0750 \mathrm{~m} / \mathrm{z}$, Found. $[\mathrm{M}+\mathrm{H}]^{+}: 328.0750 \mathrm{~m} / \mathrm{z}$

\subsection{Synthesis of 3,5-difluoro-2,4,6-tris(4-(phenylethynyl)phenyl)pyridine (1a)}

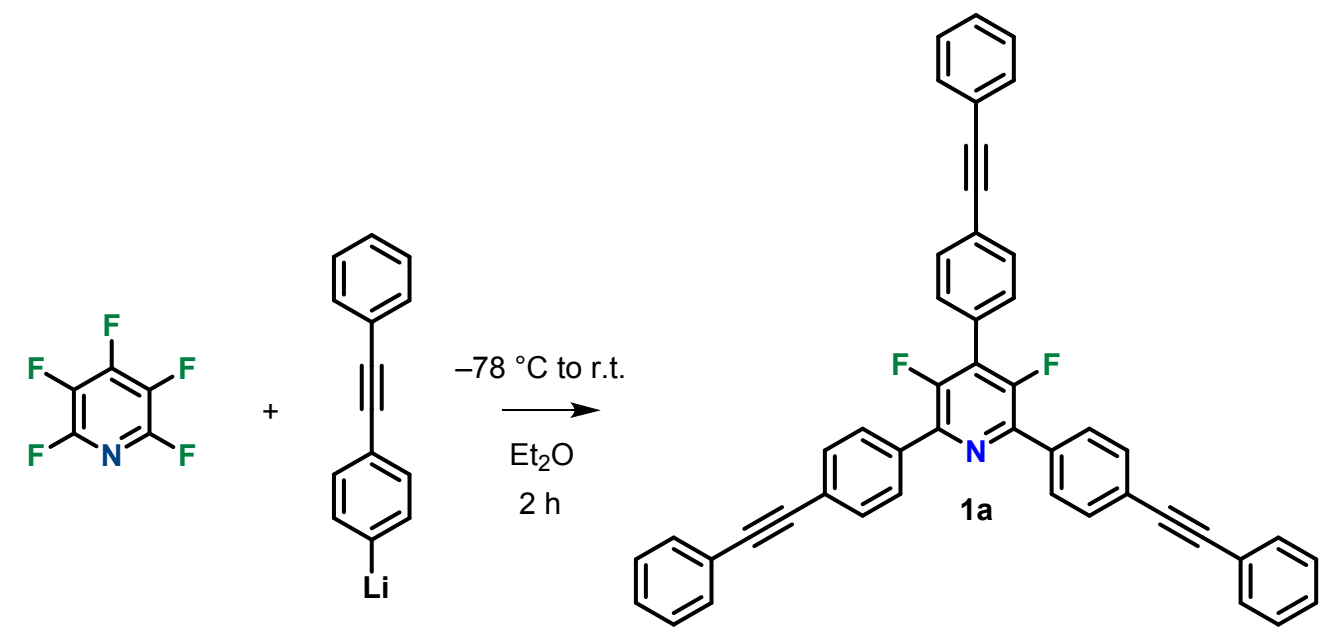

Perfluoropyridine $(0.3025 \mathrm{~g}, 1.789 \mathrm{mmol})$ dissolved in diethyl ether $(15 \mathrm{~mL})$ was added dropwise to lithiated diphenylacetylene (generated from $1.380 \mathrm{~g}, 5.368 \mathrm{mmol}$ of 1-bromo-4(phenylethynyl)benzene) in diethyl ether $(30 \mathrm{~mL}, 0.1789 \mathrm{M})$ at $-78{ }^{\circ} \mathrm{C}$ over a period of $10 \mathrm{~min}$. The reaction mixture was allowed to warm to room temperature and was stirred for $2 \mathrm{~h}$ where upon the white slurry was quenched by dropwise addition of methanol $(5 \mathrm{~mL})$ followed by the addition of aqueous ammonium chloride. The top ether layer was extracted, dried over magnesium sulfate, and concentrated under reduced pressure to give a red viscous oil. The crude product was passed through a silica plug using hexanes/ethyl acetate $(75: 25, \mathrm{v} / \mathrm{v})$ as the eluent and upon rotary evaporation afforded the title compound as a light yellow, waxy solid. X-ray quality crystalline solid was obtained by dissolving the crude oil in diethyl ether and layering the solution on top of deionized water. Crystals began to form at the interface of the biphasic mixture 
within $1 \mathrm{~h}$ and after $24 \mathrm{~h}$, the desired product had fully crystallized, yielding large white crystalline shards which were collected by gravity filtration $(0.8354 \mathrm{~g}, 78 \%) .{ }^{1} \mathrm{H}$ NMR $(500 \mathrm{MHz}$, chloroformd) $\delta 8.09(\mathrm{~d}, J=8.1 \mathrm{~Hz}, 2 \mathrm{H}), 7.69(\mathrm{dd}, J=8.5,8.1 \mathrm{~Hz}, 6 \mathrm{H}), 7.58(\mathrm{dd}, J=5.2 \mathrm{~Hz}, 9 \mathrm{H}), 7.38(\mathrm{dd}, J=5.2$ $\mathrm{Hz}, 10 \mathrm{H})(27 \mathrm{H}) ;{ }^{19} \mathrm{~F}$ NMR $\left(471 \mathrm{MHz}\right.$, chloroform-d) $\delta-124.43(\mathrm{~s}, 2 \mathrm{~F}) ;{ }^{13} \mathrm{C}$ NMR $(126 \mathrm{MHz}$, chloroform-d) $\delta 154.11,151.98,141.81-140.85$ (dd, $5.9 \mathrm{~Hz}), 134.78,131.80$ (d, J = 3.3 Hz), $130.28,128.81(\mathrm{t}, J=3.5 \mathrm{~Hz}), 128.69,128.60-128.43(\mathrm{~m}), 127.23,124.76,124.33,123.19$, 123.01, 91.12, 90.98, 89.31, 88.84; HRMS (ESI) calcd. for $\mathrm{C}_{47} \mathrm{H}_{28} \mathrm{~F}_{2} \mathrm{~N}[\mathrm{M}+\mathrm{H}]^{+}: 644.2190 \mathrm{~m} / \mathrm{z}$, Found $[\mathrm{M}+\mathrm{H}]^{+}: 644.2189 \mathrm{~m} / \mathrm{z}$.

\subsection{Synthesis of 3,5-difluoro-2,6-bis(naphthalen-1-yloxy)-4-(4(phenylethynyl)phenyl)-} pyridine (1b)
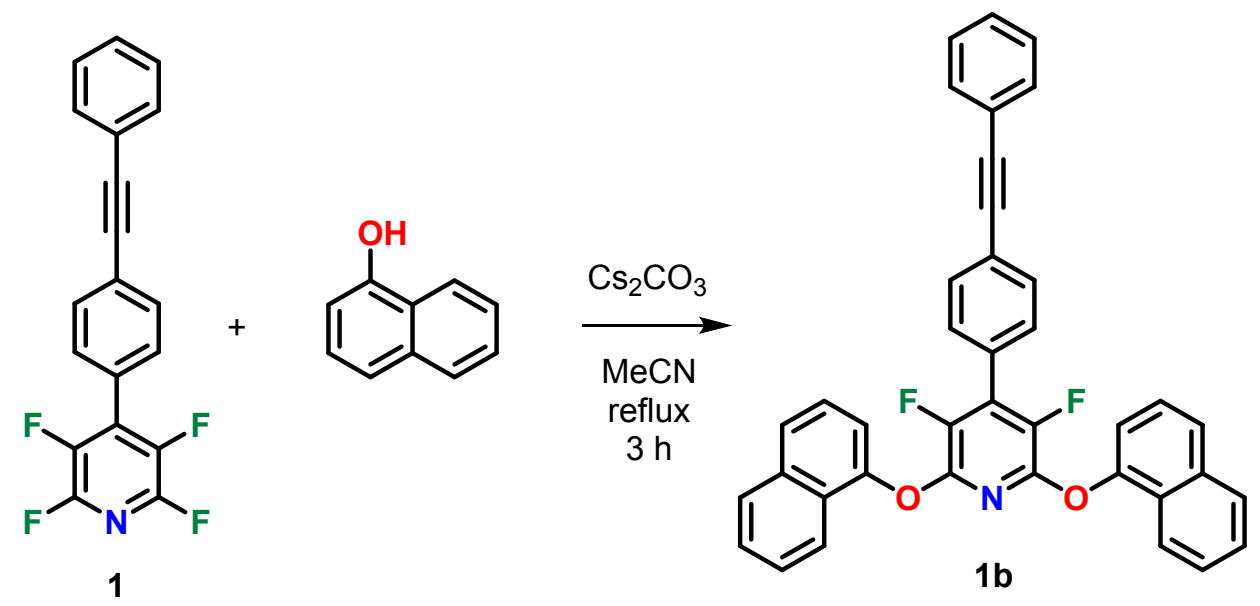

Naphthalen-1-ol $(0.3140 \mathrm{~g}, 2.178 \mathrm{mmol})$ and cesium carbonate $(0.7356 \mathrm{~g}, 2.258 \mathrm{mmol})$ were added to 2,3,5,6-tetrafluoro-4-(4-(phenylethynyl)phenyl)pyridine (1) $(0.3564 \mathrm{~g}, 1.089 \mathrm{mmol})$ dissolved in acetonitrile $(45 \mathrm{~mL})$. The reaction mixture was heated to reflux at which point the reaction mixture immediately turned a light green color. After $3 \mathrm{~h}$, the reaction mixture was cooled, vacuum filtered to remove the residual cesium salts, dried under reduced pressure to 
give a dark solid which was triturated repeatedly with methanol to give a white solid as the desired product $(0.3770 \mathrm{~g}, 60 \%) .{ }^{1} \mathrm{H}$ NMR $(500 \mathrm{MHz}$, chloroform-d) $\delta 7.72(\mathrm{t}, \mathrm{J}=6.9 \mathrm{~Hz}, 4 \mathrm{H}), 7.60$ (dt, $J=10.4,9.1,5.3 \mathrm{~Hz}, 6 \mathrm{H}), 7.50(\mathrm{~d}, J=1.4 \mathrm{~Hz}, 2 \mathrm{H}), 7.42(\mathrm{t}, J=8 \mathrm{~Hz}, 3 \mathrm{H}), 7.38(\mathrm{dd}, \mathrm{J}=5.3 \mathrm{~Hz}, 6 \mathrm{H})$, $7.21(\mathrm{dd}, J=8.7,2 \mathrm{~Hz}, 2 \mathrm{H})(23 \mathrm{H}) ;{ }^{19} \mathrm{~F} \mathrm{NMR}\left(471 \mathrm{MHz}\right.$, chloroform-d) $\delta-146.16(\mathrm{~s}, 2 \mathrm{~F}) ;{ }^{13} \mathrm{C}$ NMR (126 MHz, chloroform-d) $\delta 151.38,144.46(\mathrm{~d}, J=14.3 \mathrm{~Hz}), 140.73,138.68,133.85,131.85(\mathrm{~d}, J=$ $5.3 \mathrm{~Hz}), 130.87,130.15,129.23,128.70,128.52,127.70,127.46,127.22,126.40,125.21,125.01$, 123.00, 120.87, 116.86, 91.23, 88.81; HRMS (ESI) calcd. for $\mathrm{C}_{39} \mathrm{H}_{23} \mathrm{~F}_{2} \mathrm{NO}_{2} \mathrm{M}^{+}: 575.1697 \mathrm{~m} / \mathrm{z}$, Found. $\mathrm{M}^{+}: 575.1692 \mathrm{~m} / \mathrm{z}$.

CCDC contains the supplementary crystallographic data for this paper for compound $\mathbf{1} \mathbf{b}$. The CCDC reference number is 1987229 . These data can be viewed free of charge via http://www.ccdc.cam.ac.uk/cont/retrieving.html or from the CCDC, 12 Union Road, Cambridge CB21EZ, UK. Fax: +44 1223 336033. E-mail: deposit@ccdc.cam.ac.uk.

\subsection{Synthesis of 4-(anthracen-9-yl)-2,3,5,6-tetrafluoropyridine (2)}<smiles>Fc1nc(F)c(F)c(F)c1F</smiles><smiles>[C+]1CCCCC1</smiles><smiles>Clc1c2ccccc2cc2ccccc12</smiles><smiles>Fc1nc(F)c(F)c(-c2c3ccccc3cc3ccccc23)c1F</smiles>

2

Lithiated anthracene (generated from $1.521 \mathrm{~g}, 5.915 \mathrm{mmol}$ of 9-bromoanthracene) in diethyl ether (30 mL, $0.1972 \mathrm{M})$ at $-78^{\circ} \mathrm{C}$ was added dropwise to perfluoropyridine $(1.009 \mathrm{~g}, 5.968 \mathrm{mmol})$ dissolved in diethyl ether $(15 \mathrm{~mL})$ at $-78{ }^{\circ} \mathrm{C}$ over a period of $10 \mathrm{~min}$. Upon initial addition of the lithiated anthracene, the reaction mixture turned orange. The reaction mixture was allowed to warm to room temperature during which time the reaction mixture turned a light brown. After 
$2 \mathrm{~h}$, the reaction was quenched by the addition of methanol $(5 \mathrm{~mL})$ followed by the addition of aqueous ammonium chloride. The top ether layer was extracted, dried over magnesium sulfate, and then concentrated under reduced pressure to afford a red viscous oil. The crude product was passed through a silica gel flash column using hexanes/ethyl acetate $(3: 1, \mathrm{v} / \mathrm{v})$ as the eluent. The collected fractions were dried to give the desired product as a light yellow solid (1.038 g, 53\%). ${ }^{1} \mathrm{H}$ NMR $\left(500 \mathrm{MHz}\right.$, chloroform-d) $\delta 8.69(\mathrm{~s}, 1 \mathrm{H}), 8.13(\mathrm{~d}, 8.1 \mathrm{~Hz}, 2 \mathrm{H}), 7.60-7.45(\mathrm{~m}, 6 \mathrm{H})(9 \mathrm{H}) ;{ }^{19} \mathrm{~F}$ $\operatorname{NMR}\left(471 \mathrm{MHz}\right.$, chloroform-d) $\delta$-89.64-(-90.00) (m, 2F), -139.12-(-139.48) (m, 2F); ${ }^{13} \mathrm{C}$ NMR (126 MHz, Chloroform-d) $\delta 144.99$ (t, $12.7 \mathrm{~Hz}), 143.02$ (t, $16.7 \mathrm{~Hz}), 141.51$ (dd, $27.1 \mathrm{~Hz}$ ), 139.45 (dd, $27 \mathrm{~Hz}), 131.22,130.40,129.67,129.19,127.66,125.78,124.22,118.81$. HRMS (ESI) calcd. for $\mathrm{C}_{19} \mathrm{H}_{9} \mathrm{~F}_{4} \mathrm{~N} \mathrm{M}^{+}: 327.0671 \mathrm{~m} / \mathrm{z}$, Found $\mathrm{M}^{+}: 327.0672 \mathrm{~m} / \mathrm{z}$.

\subsection{Synthesis of 2-(anthracen-9-yl)-3,5-difluoro-4,6-bis(4-(phenylethynyl)phenyl)pyridine}

(2a)

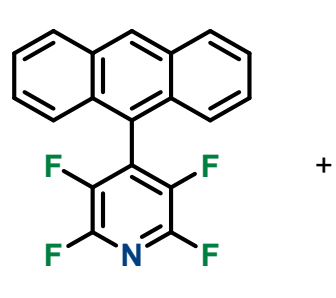

2

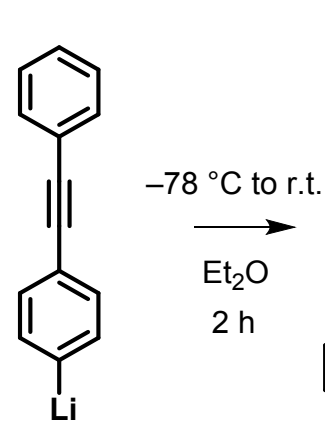

$\mathrm{h}$

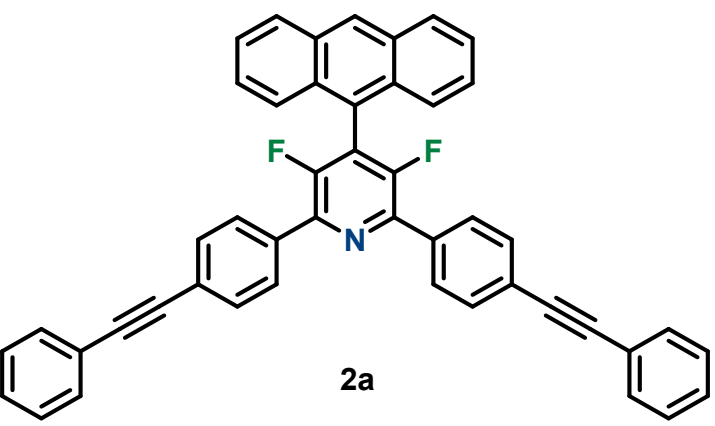

Lithiated diphenylacetylene (generated from $0.4289 \mathrm{~g}, 1.668 \mathrm{mmol}$ of 1-bromo-4(phenylethynyl)benzene) in diethyl ether $(30 \mathrm{~mL}, 0.0556 \mathrm{M})$ at $-78^{\circ} \mathrm{C}$ was added dropwise to 4 (anthracen-9-yl)-2,3,5,6-tetrafluoropyridine (2) $(0.2730 \mathrm{~g}, 0.8341 \mathrm{mmol}$ ) dissolved in diethyl ether $(15 \mathrm{~mL})$ at $-78{ }^{\circ} \mathrm{C}$ over a period of $10 \mathrm{~min}$. Upon initial addition of lithiated diphenylacetylene, the reaction mixture turned a deep red/purple. The reaction mixture was 
allowed to warm to room temperature during which time the reaction mixture turned a bright yellow. After $2 \mathrm{~h}$, the reaction was quenched, first by the addition of methanol $(5 \mathrm{~mL})$, followed by the addition of aqueous ammonium chloride. The top ether layer was extracted, dried over magnesium sulfate, and then concentrated under reduced pressure to give a red viscous oil. The crude product was passed through a silica gel flash column using hexanes/ethyl acetate (3:1, v/v) as the eluent. The collected fractions were vacuum dried to give the desired product as a light orange solid (0.3382 g, 63\%). ${ }^{1} \mathrm{H}$ NMR (500 MHz, chloroform-d) $\delta 8.68(\mathrm{~s}, 1 \mathrm{H}), 8.13(\mathrm{~d}, \mathrm{~J}=8.3 \mathrm{~Hz}$, $3 \mathrm{H}), 7.70(\mathrm{td}, \mathrm{J}=7.3 \mathrm{~Hz}, 4 \mathrm{H}), 7.60-7.44(\mathrm{~m}, 10 \mathrm{H}), 7.37(\mathrm{td}, \mathrm{J}=6.7,1.2 \mathrm{~Hz}, 9 \mathrm{H})(27 \mathrm{H}) ;{ }^{19} \mathrm{~F} \mathrm{NMR}(471$ $\mathrm{MHz}$, chloroform-d) $\delta-119.67(\mathrm{~s}, 2 \mathrm{~F}) ;{ }^{13} \mathrm{C}$ NMR $\left(126 \mathrm{MHz}\right.$, chloroform-d) ${ }^{13} \mathrm{C} \mathrm{NMR}(126 \mathrm{MHz}$, chloroform-d) $\delta$ 155.51, 153.37, 141.09 (d, $J=10.6 \mathrm{~Hz}), 134.72,131.83$ (d, J = 11.2 Hz), 131.37, $130.05,129.39,129.05,128.86,128.56,128.49,127.11,125.63,125.03,124.44,123.18,121.31$, 91.06, 89.32; HRMS (ESI) calcd. for $\mathrm{C}_{47} \mathrm{H}_{28} \mathrm{~F}_{2} \mathrm{~N}[\mathrm{M}+\mathrm{H}]^{+}: 644.2190 \mathrm{~m} / \mathrm{z}$, Found $[\mathrm{M}+\mathrm{H}]^{+}: 644.2186$ $\mathrm{m} / \mathrm{z}$.

\subsection{Synthesis of 2,4,6-tri(anthracen-9-yl)-3,5-difluoropyridine (2b)}<smiles>Fc1nc(F)c(F)c(F)c1F</smiles><smiles>Clc1c2ccccc2cc2ccccc12</smiles>

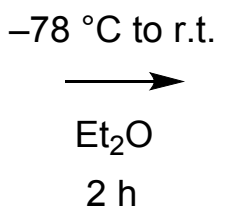<smiles>Fc1c(-c2c3ccccc3cc3ccccc23)nc(-c2c3ccccc3cc3ccccc23)c(F)c1-c1c2ccccc2cc2ccccc12</smiles>

Lithiated diphenylacetylene (generated from $0.9176 \mathrm{~g}, 3.569 \mathrm{mmol}$ of 1-bromo-4(phenylethynyl)benzene) in diethyl ether $(30 \mathrm{~mL}, 0.1189 \mathrm{M})$ at $-78{ }^{\circ} \mathrm{C}$ was then added dropwise to perfluoropyridine $(0.2008 \mathrm{~g}, 1.188 \mathrm{mmol})$ dissolved in diethyl ether $(15 \mathrm{~mL})$ at $-78{ }^{\circ} \mathrm{C}$ over a 
period of $10 \mathrm{~min}$. Upon initial addition of the lithiated diphenylacetylene, the reaction mixture turned a deep red/purple. The reaction mixture was allowed to warm to room temperature during which time the reaction mixture turned a bright yellow. After $2 \mathrm{~h}$, the reaction was quenched, first by the addition of methanol $(5 \mathrm{~mL})$, followed by the addition of aqueous ammonium chloride. The top ether layer was extracted, dried over magnesium sulfate, and then concentrated under reduced pressure to give a red viscous oil. The crude product was passed through a silica gel flash column using hexanes/ethyl acetate $(3: 1, \mathrm{v} / \mathrm{v})$ as the eluent. The collected fractions were dried to give the desired product as a light yellow solid (0.6273 g, 82\%). ${ }^{1} \mathrm{H}$ NMR (500 MHz, chloroform-d) $\delta 8.66(\mathrm{~s}, 1 \mathrm{H}), 8.58(\mathrm{~s}, 3 \mathrm{H}), 8.13(\mathrm{~d}, J=8.4 \mathrm{~Hz}, 2 \mathrm{H}), 8.07(\mathrm{~d}, J=$ $8.4 \mathrm{~Hz}, 6 \mathrm{H}), 8.02(\mathrm{~d}, J=8.5 \mathrm{~Hz}, 5 \mathrm{H}), 7.68(\mathrm{td}, J=7.4,1 \mathrm{~Hz}, 2 \mathrm{H}), 7.64-7.54(\mathrm{~m}, 5 \mathrm{H}), 7.51(\mathrm{t}, J=7.4$ $\mathrm{Hz}, 3 \mathrm{H})(27 \mathrm{H}) ;{ }^{19} \mathrm{~F} N M R\left(500 \mathrm{MHz}\right.$, chloroform-d) $\delta-115.82(\mathrm{~s}, 2 \mathrm{~F}) ;{ }^{13} \mathrm{C}$ NMR $(126 \mathrm{MHz}$, chloroform-d) $\delta 157.00,154.90,143.11(\mathrm{dd}, J=16.7,6.3 \mathrm{~Hz}), 131.53,131.41,130.69,130.13$ $129.72,129.25,129.01,128.97,128.36(d, J=2.6 \mathrm{~Hz}), 127.39,126.77,125.60,125.31,125.19$, 124.77, 120.75; HRMS (ESI) calcd. for $\mathrm{C}_{47} \mathrm{H}_{28} \mathrm{~F}_{2} \mathrm{~N}[\mathrm{M}+\mathrm{H}]^{+}: 644.2190 \mathrm{~m} / \mathrm{z}$, Found $[\mathrm{M}+\mathrm{H}]^{+}: 644.2184$ $\mathrm{m} / \mathrm{z}$. 


\section{NMR Spectra}

${ }^{1} \mathrm{H}$ NMR spectra of 2,3,5,6-tetrafluoro-4-(4-(phenylethynyl)phenyl)pyridine (1)

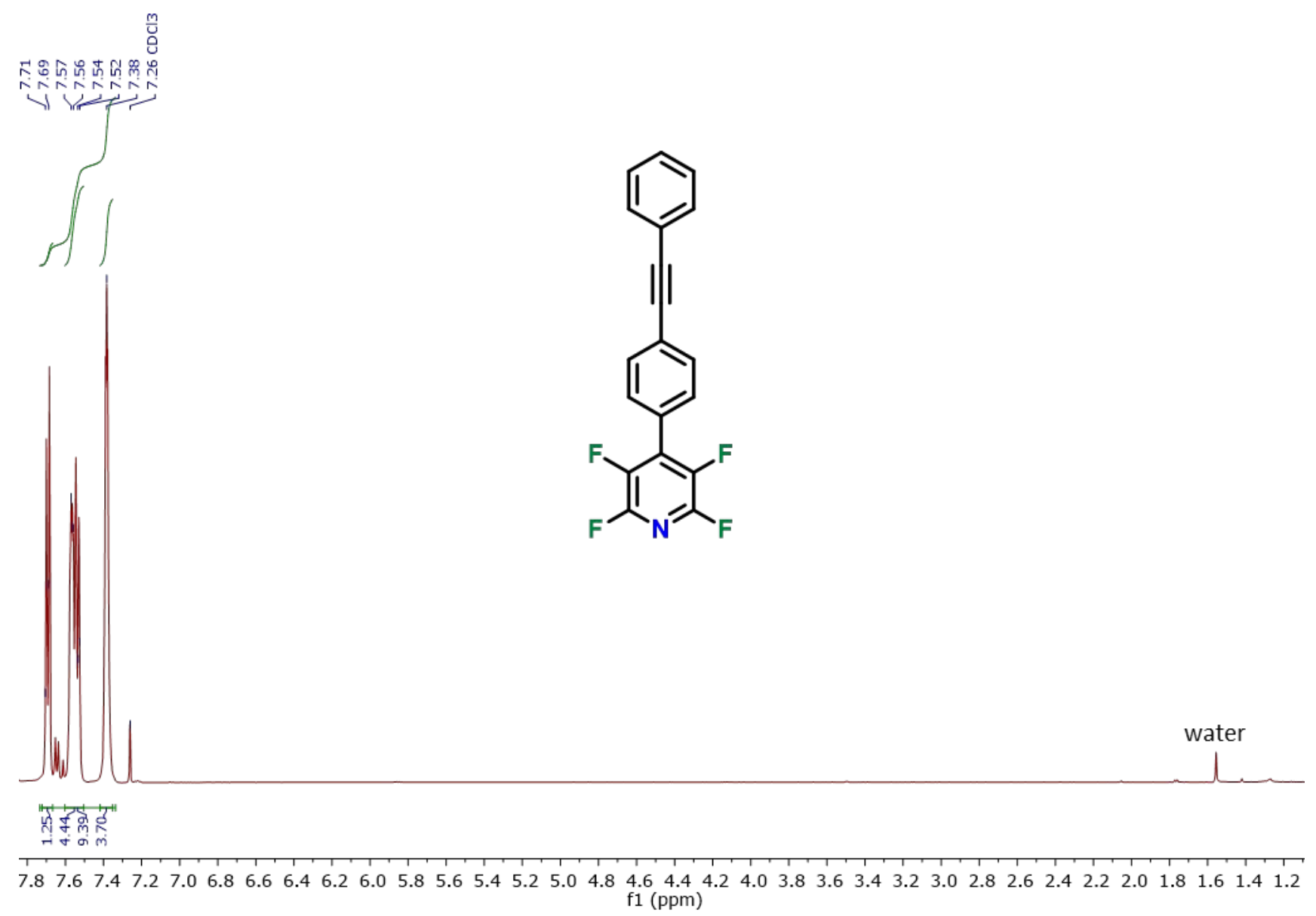


${ }^{19} \mathrm{~F}$ NMR spectra of 2,3,5,6-tetrafluoro-4-(4-(phenylethynyl)phenyl)pyridine (1)

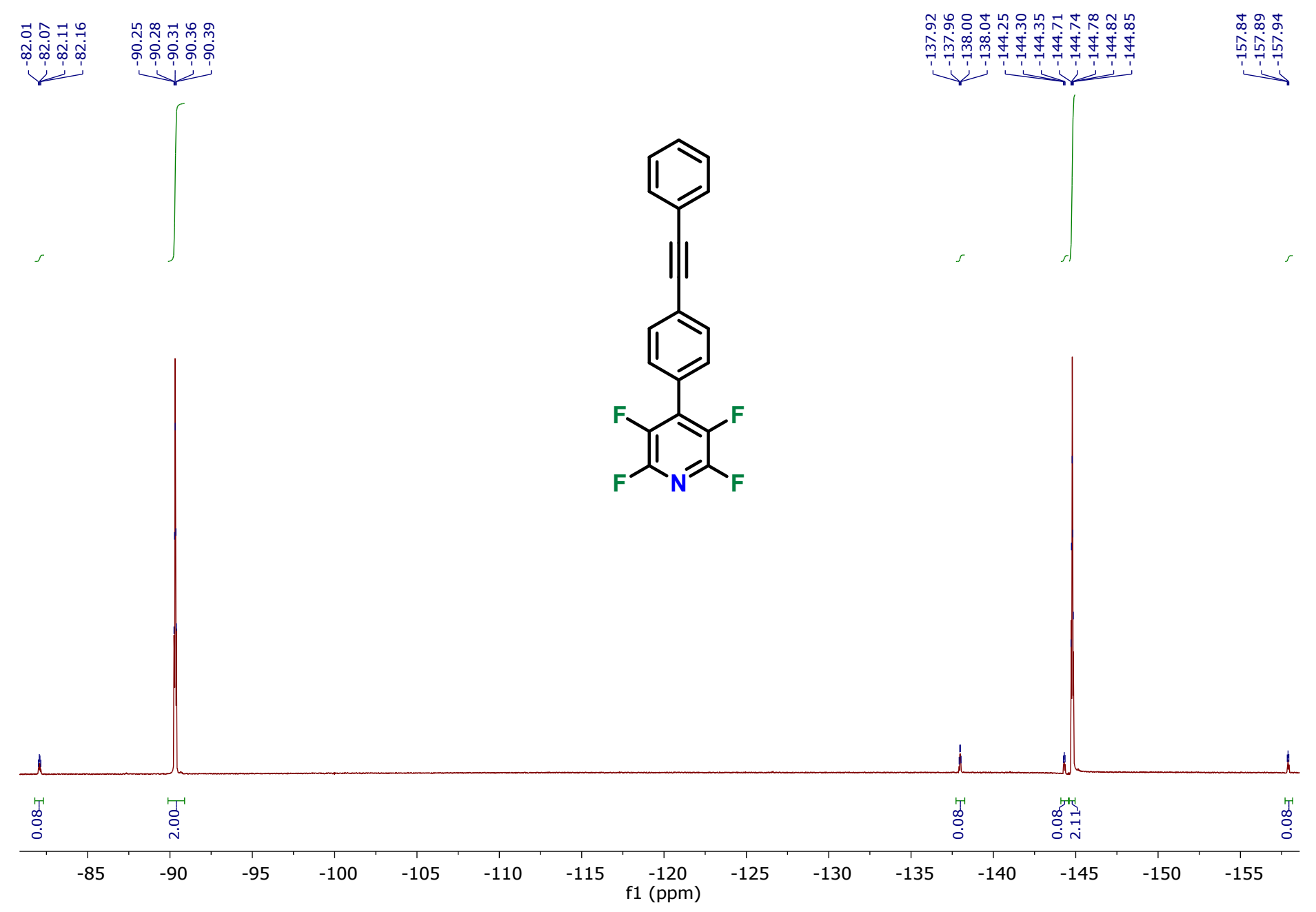


${ }^{13} \mathrm{C}$ NMR spectra of 2,3,5,6-tetrafluoro-4-(4-(phenylethynyl)phenyl)pyridine (1)

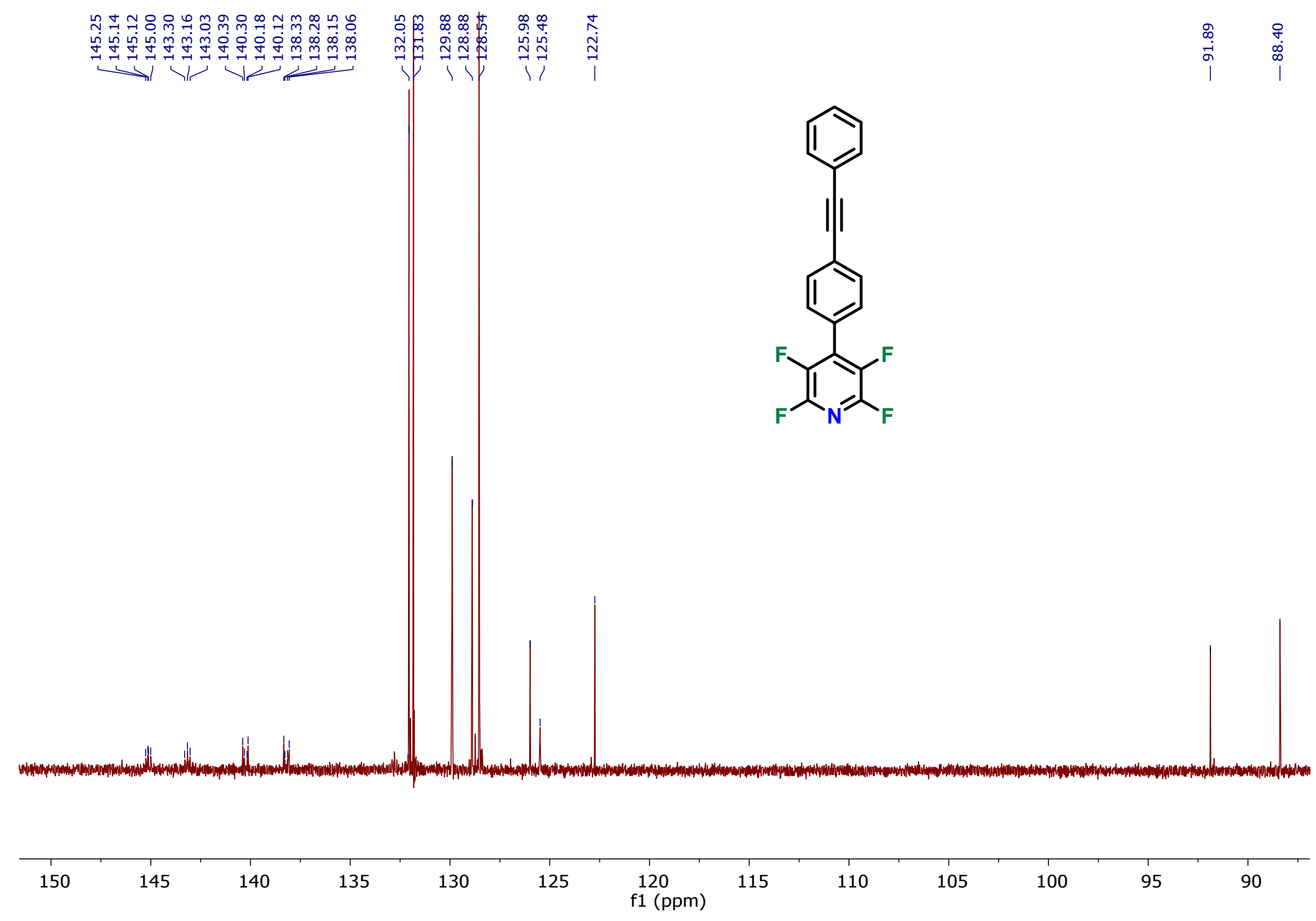


Electronic Supplementary Material (ESI) for Macro Letters.

This journal is (C) The American Chemical Society 2020

${ }^{1} \mathrm{H}$ NMR spectra of 3,5-difluoro-2,4,6-tris(4-(phenylethynyl)phenyl)pyridine (ia)

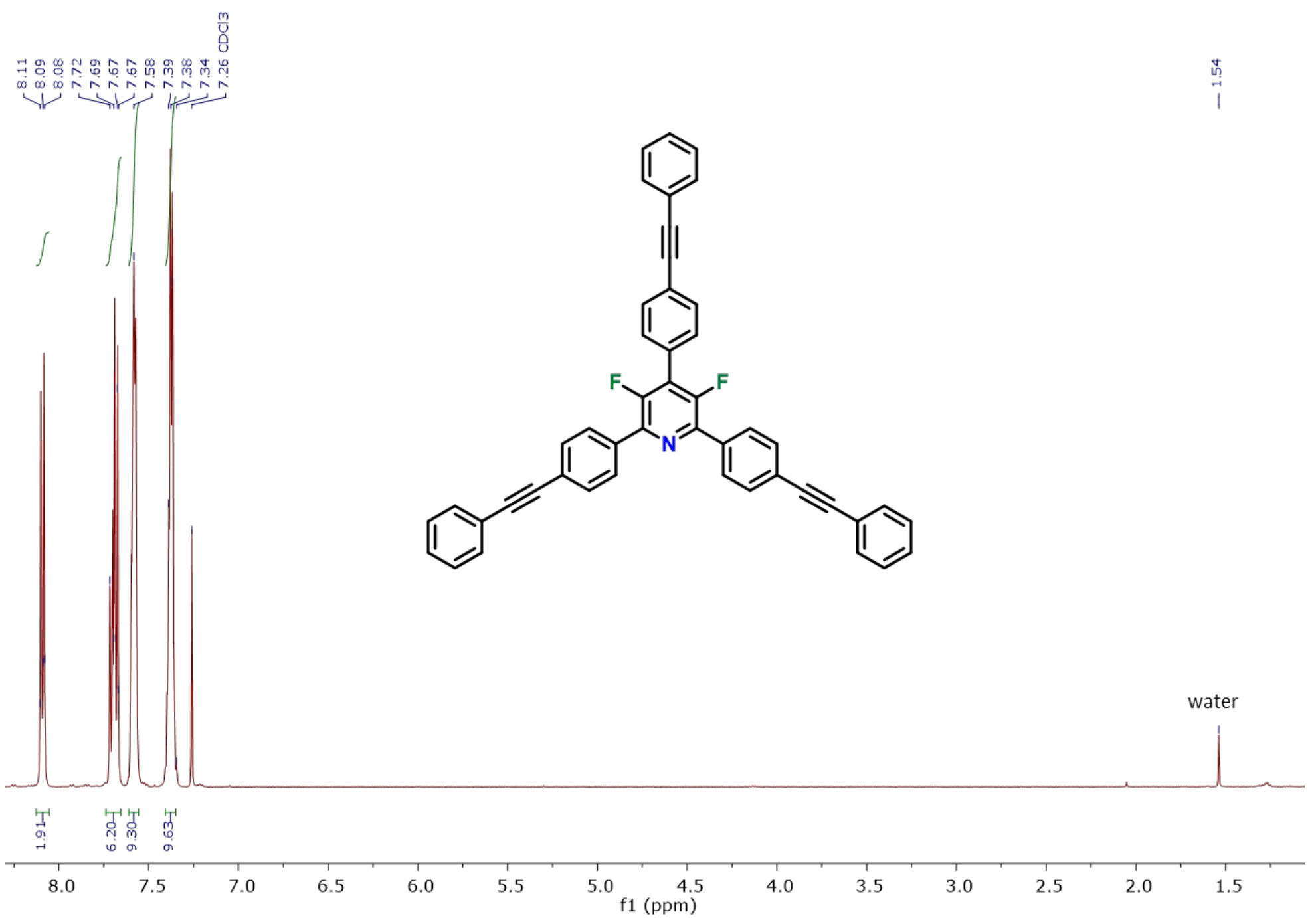

S14 
${ }^{19} \mathrm{~F}$ NMR spectra of 3,5-difluoro-2,4,6-tris(4-(phenylethynyl)phenyl)pyridine (1a)

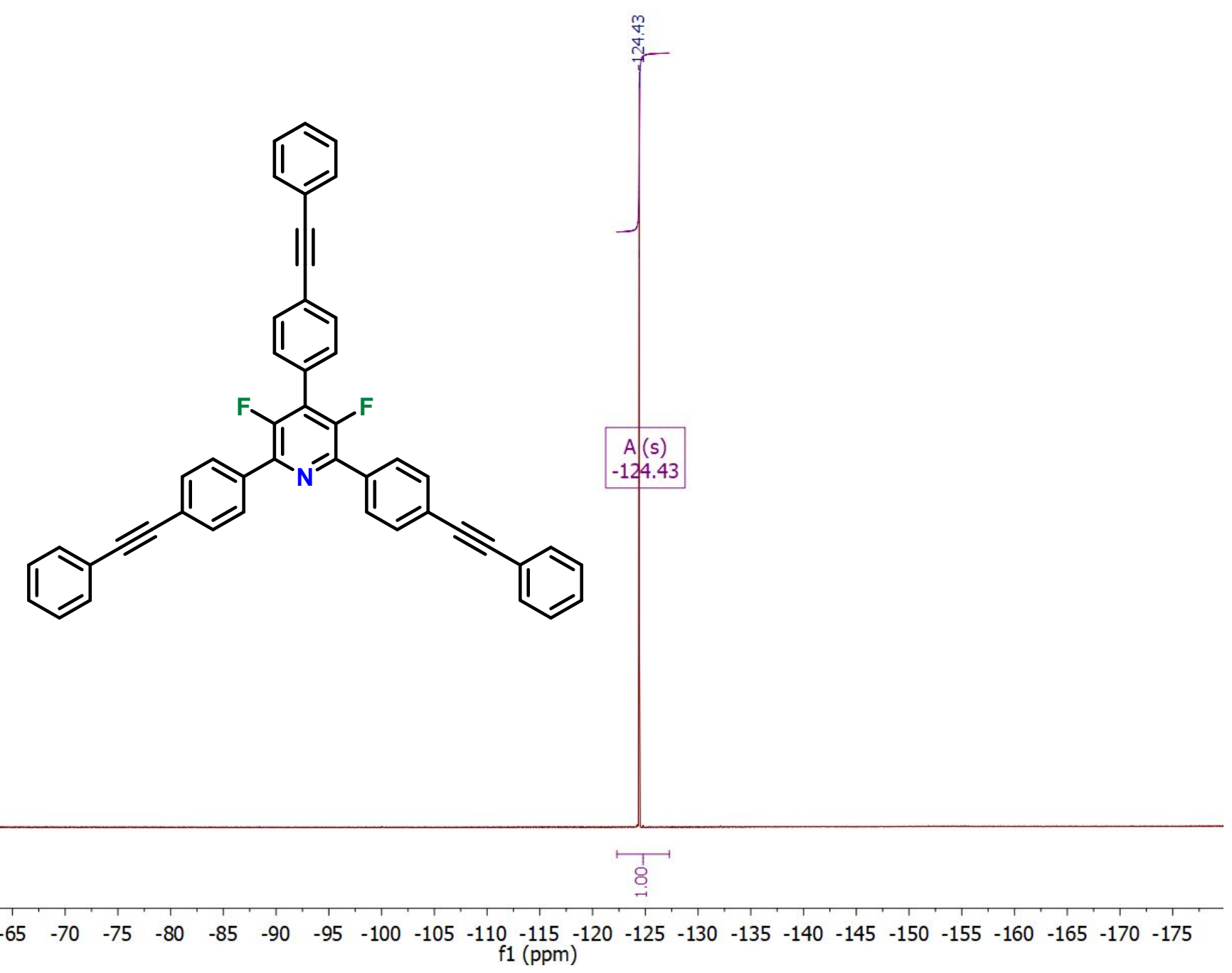


${ }^{13} \mathrm{C}$ NMR spectra of 3,5-difluoro-2,4,6-tris(4-(phenylethynyl)phenyl)pyridine (1a)

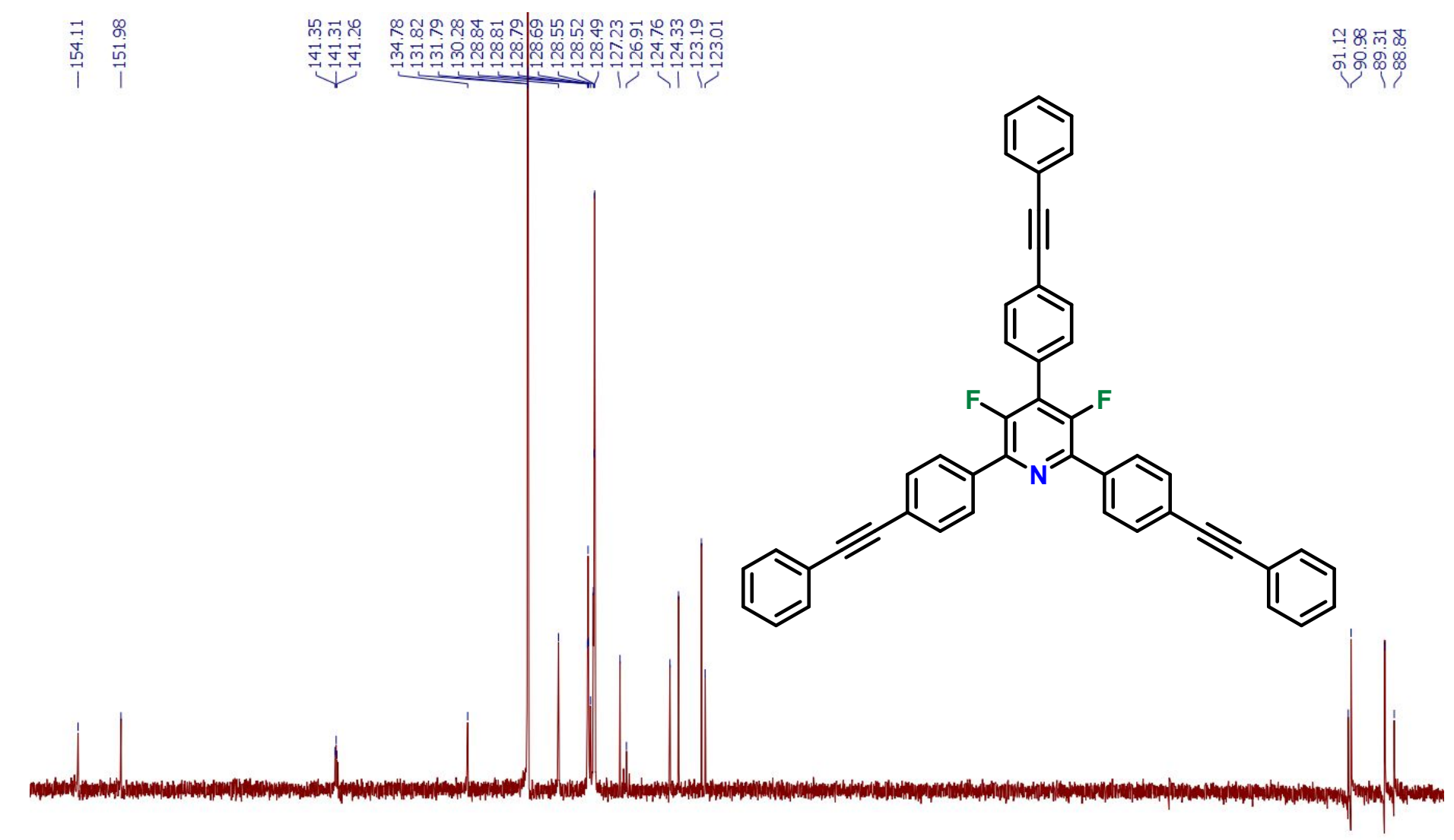

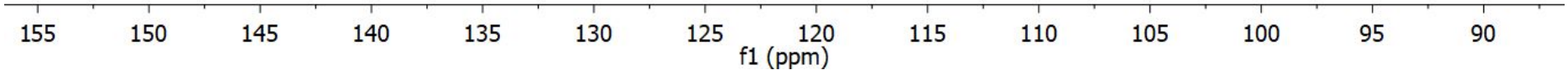




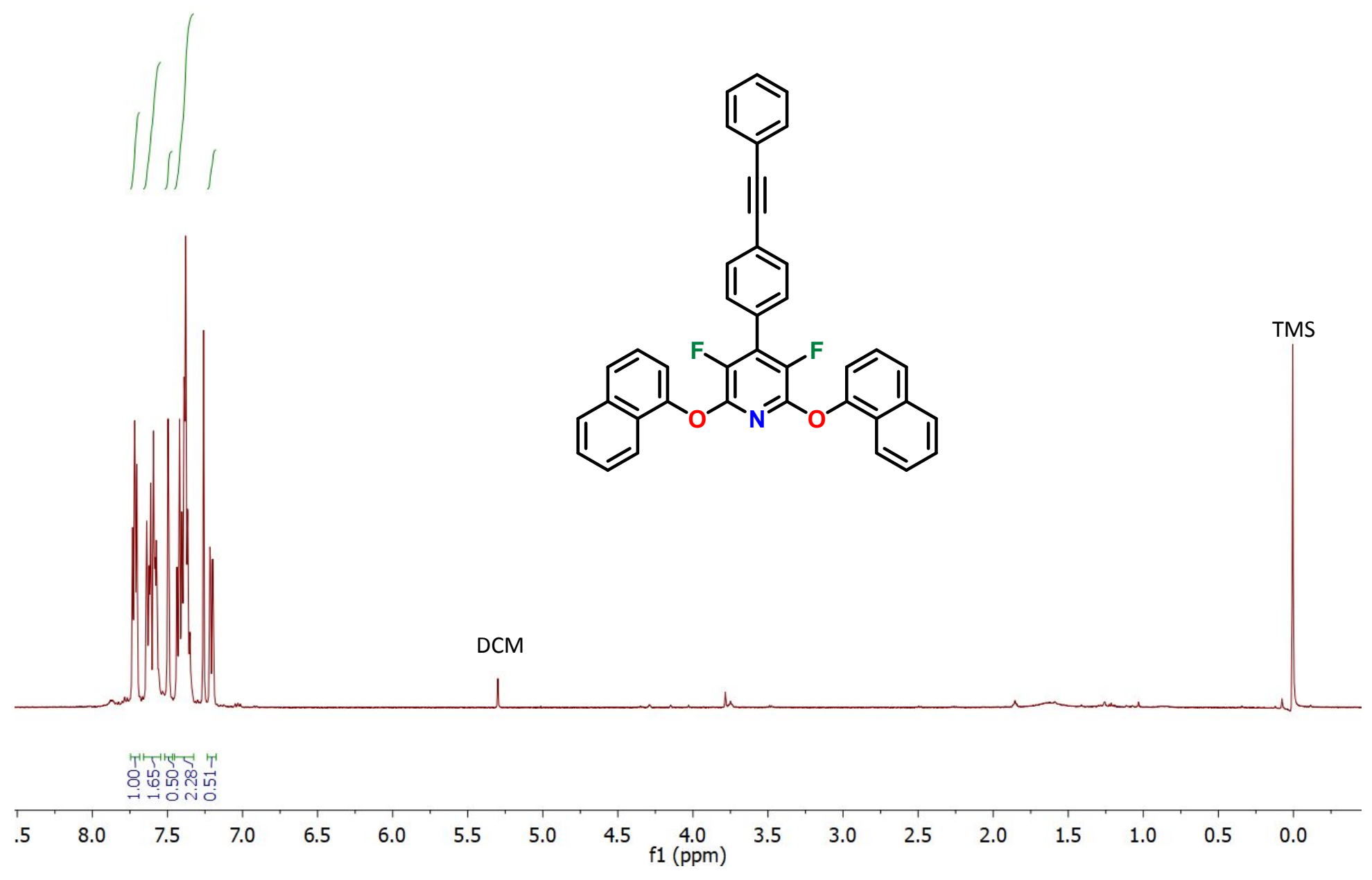


${ }^{19} \mathrm{~F}$ NMR spectra of 3,5-difluoro-2,6-bis(naphthalen-1-yloxy)-4-(4-(phenylethynyl)phenyl)pyridine (1b)

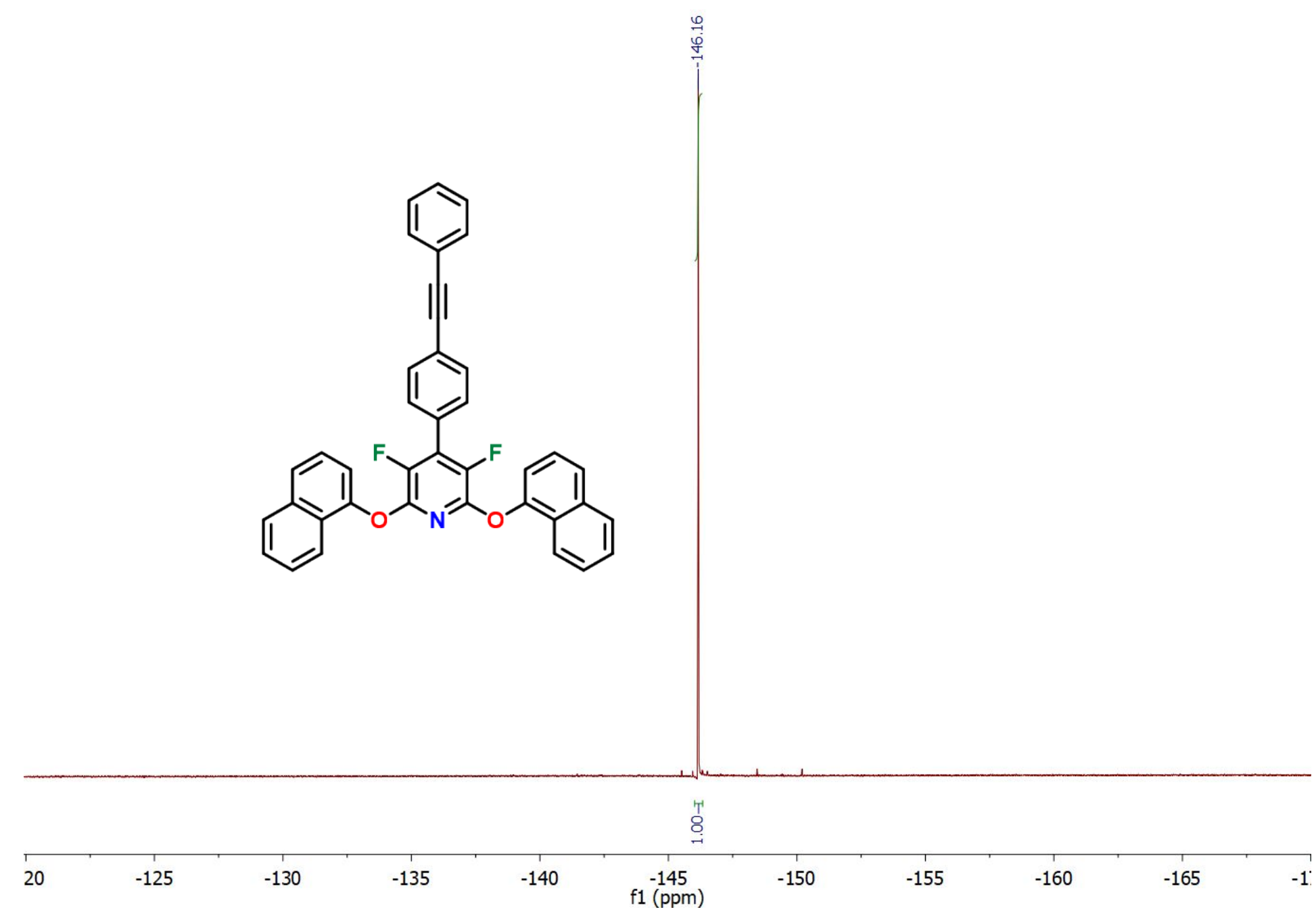




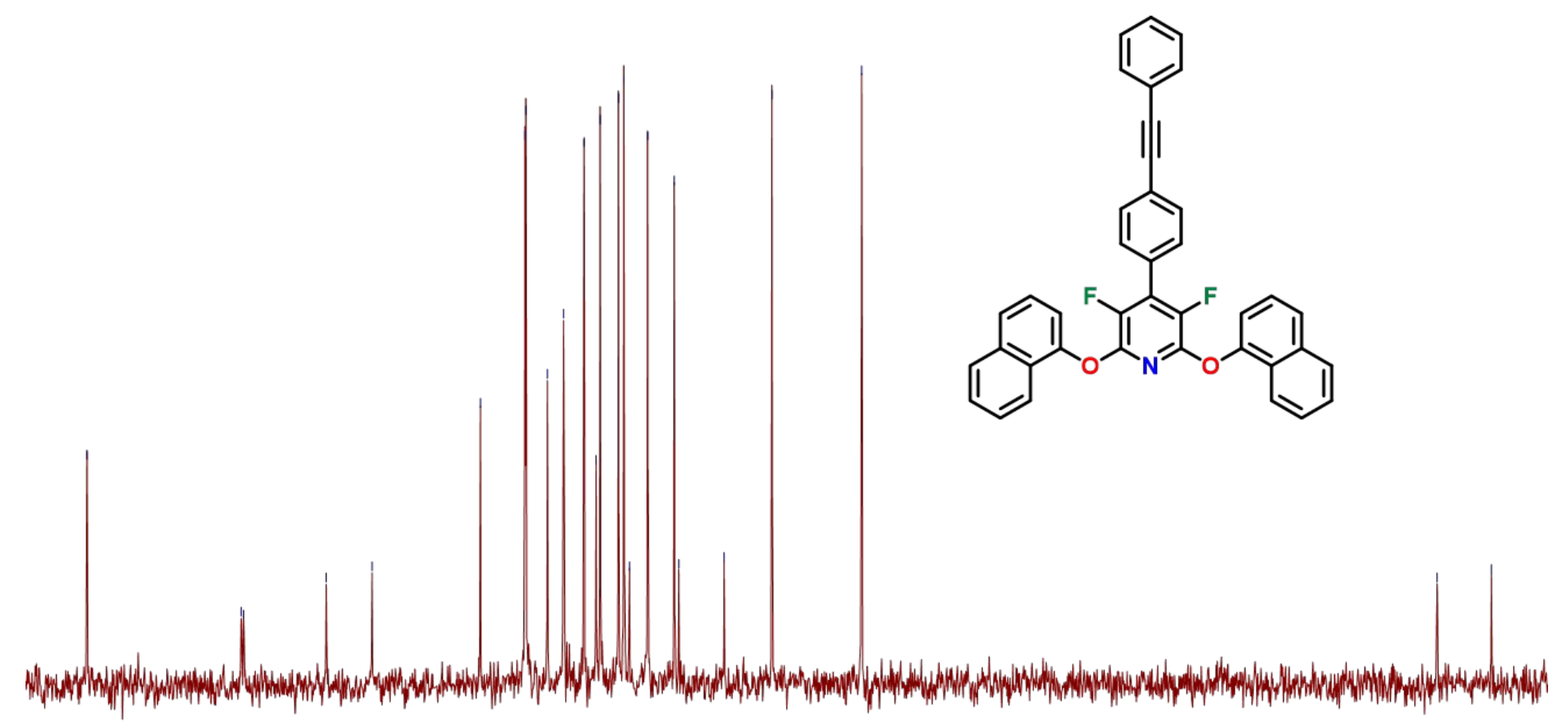

54152150148146144142140138136134132130128126124122120118116114112110108106104102100989694929088 f1 (ppm) 
${ }^{1} \mathrm{H}$ NMR spectra of 4-(anthracen-9-yl)-2,3,5,6-tetrafluoropyridine (2)

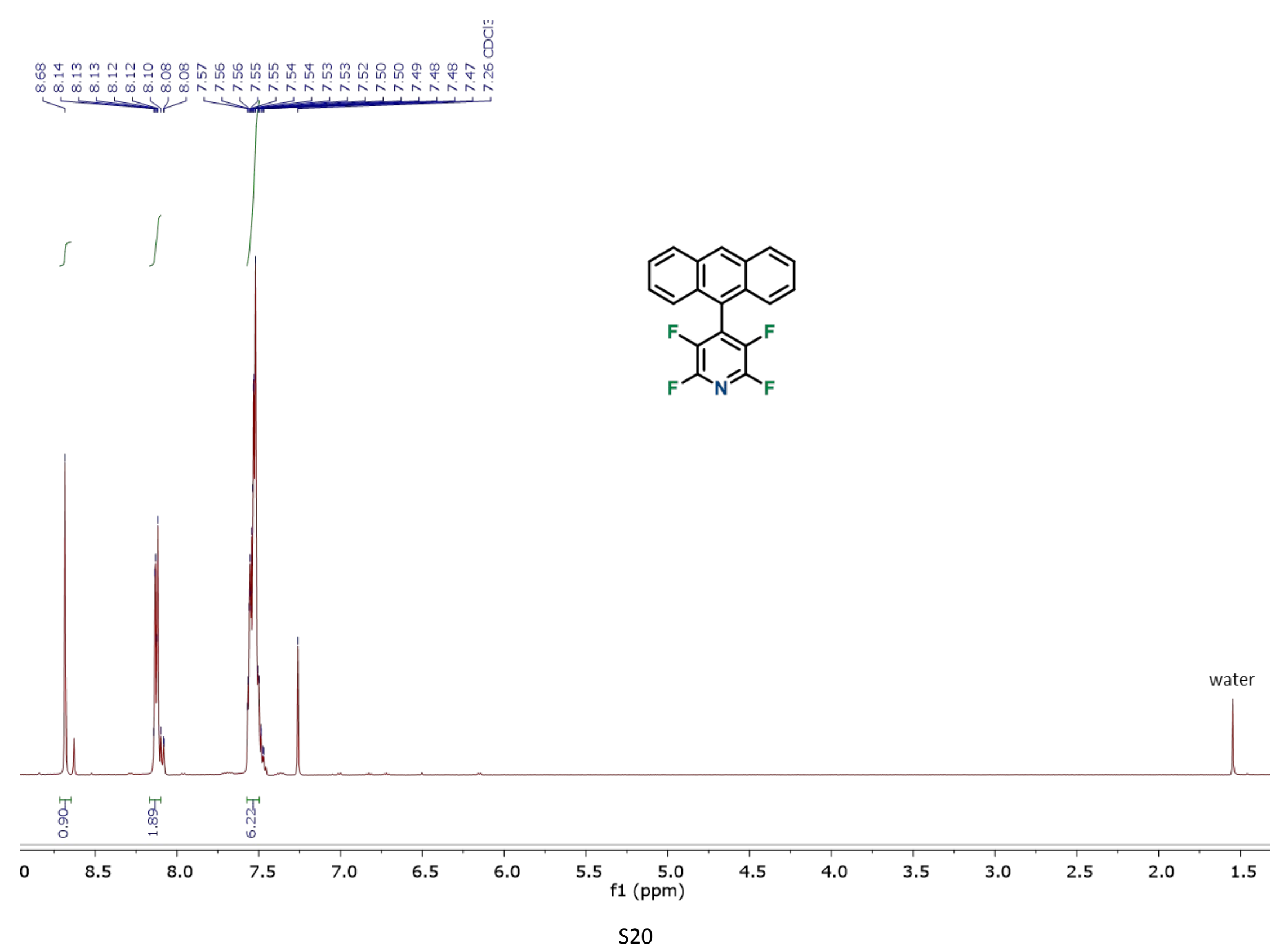


${ }^{19} \mathrm{~F}$ NMR spectra of 4-(anthracen-9-yl)-2,3,5,6-tetrafluoropyridine (2)

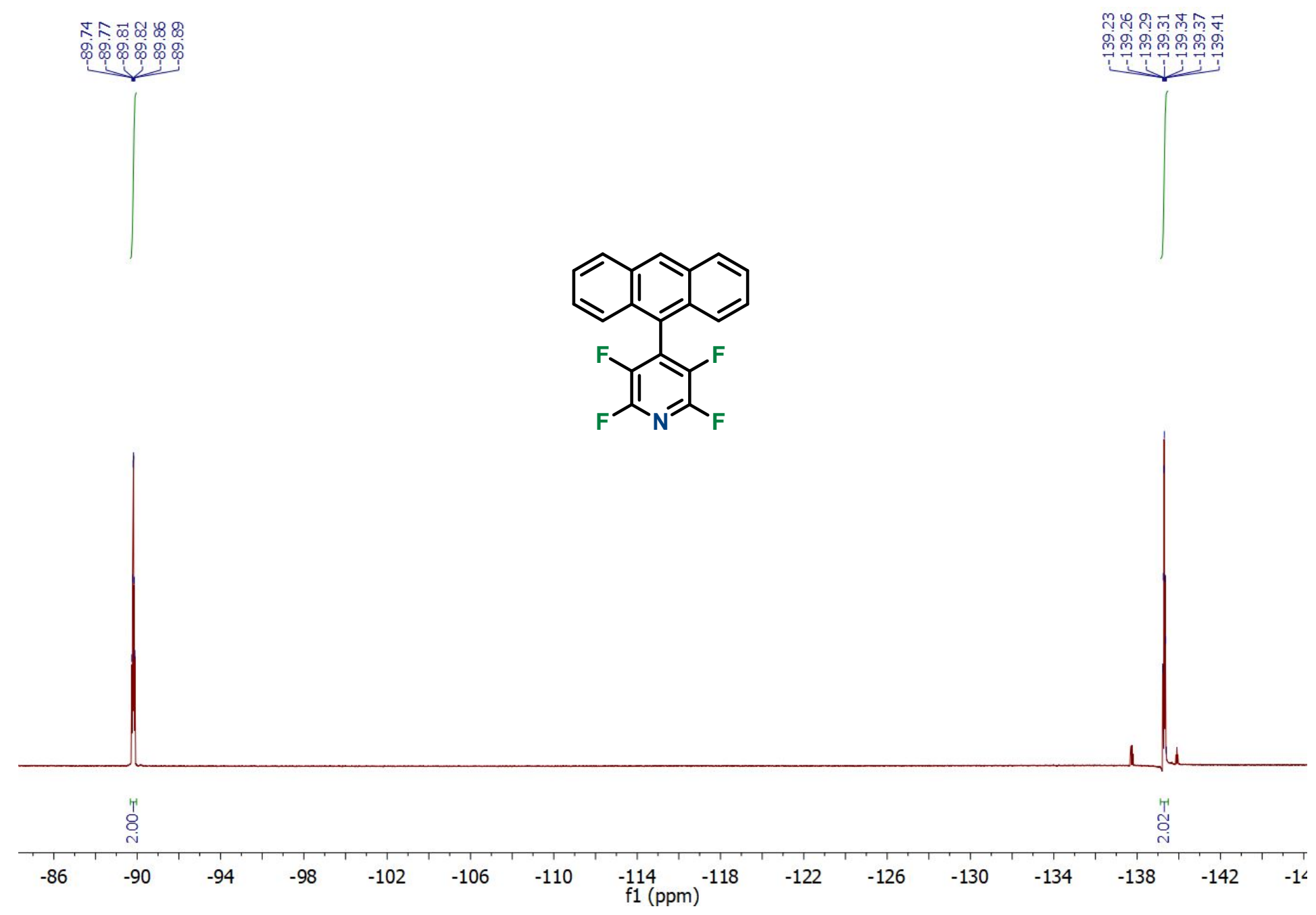




\section{${ }^{13} \mathrm{C}$ NMR spectra of 4-(anthracen-9-yl)-2,3,5,6-tetrafluoropyridine (2)}

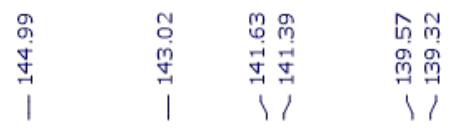

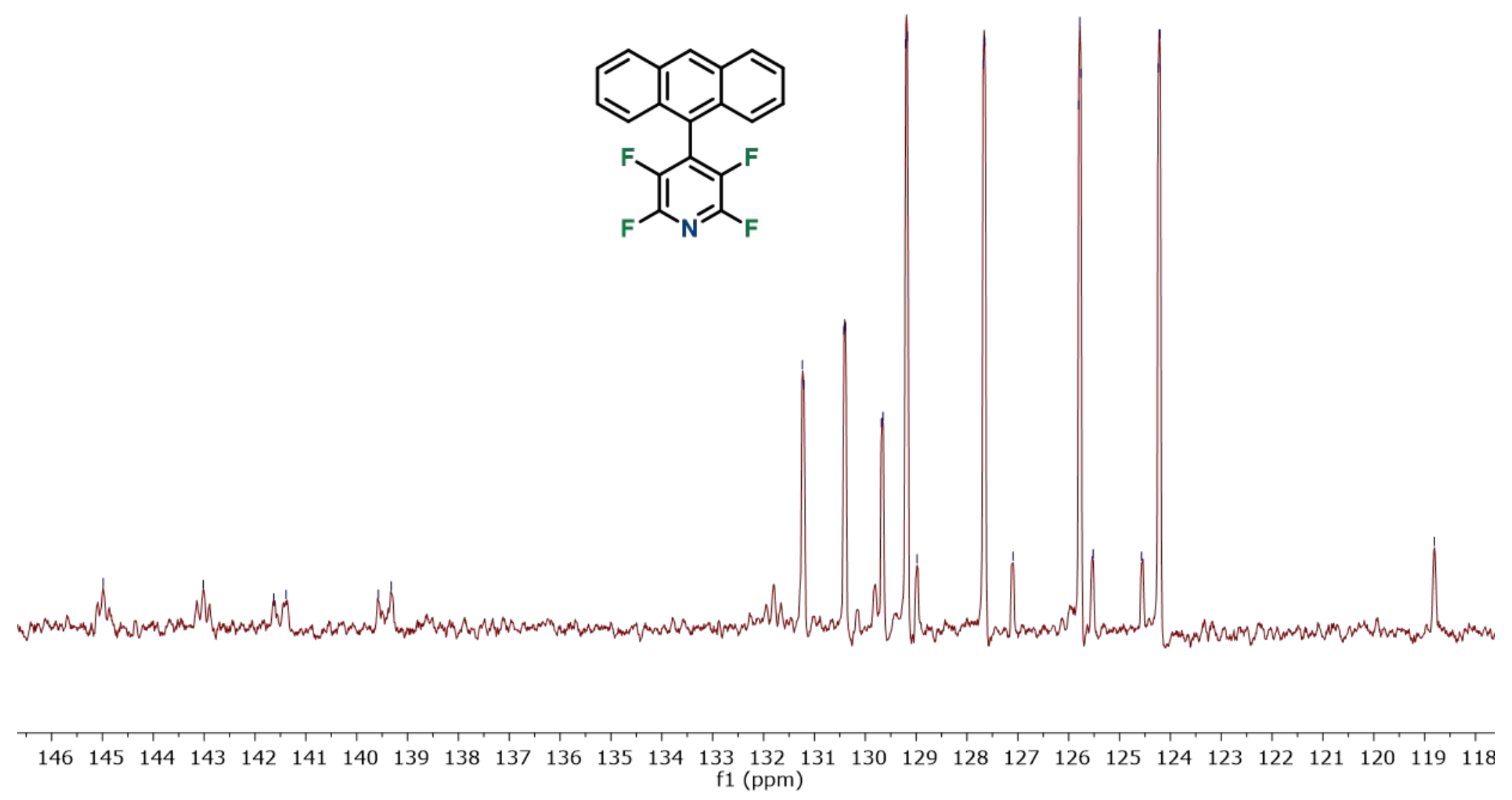


${ }^{1} \mathrm{H}$ NMR spectra of 2-(anthracen-9-yl)-3,5-difluoro-4,6-bis(4-(phenylethynyl)phenyl)pyridine (2a)

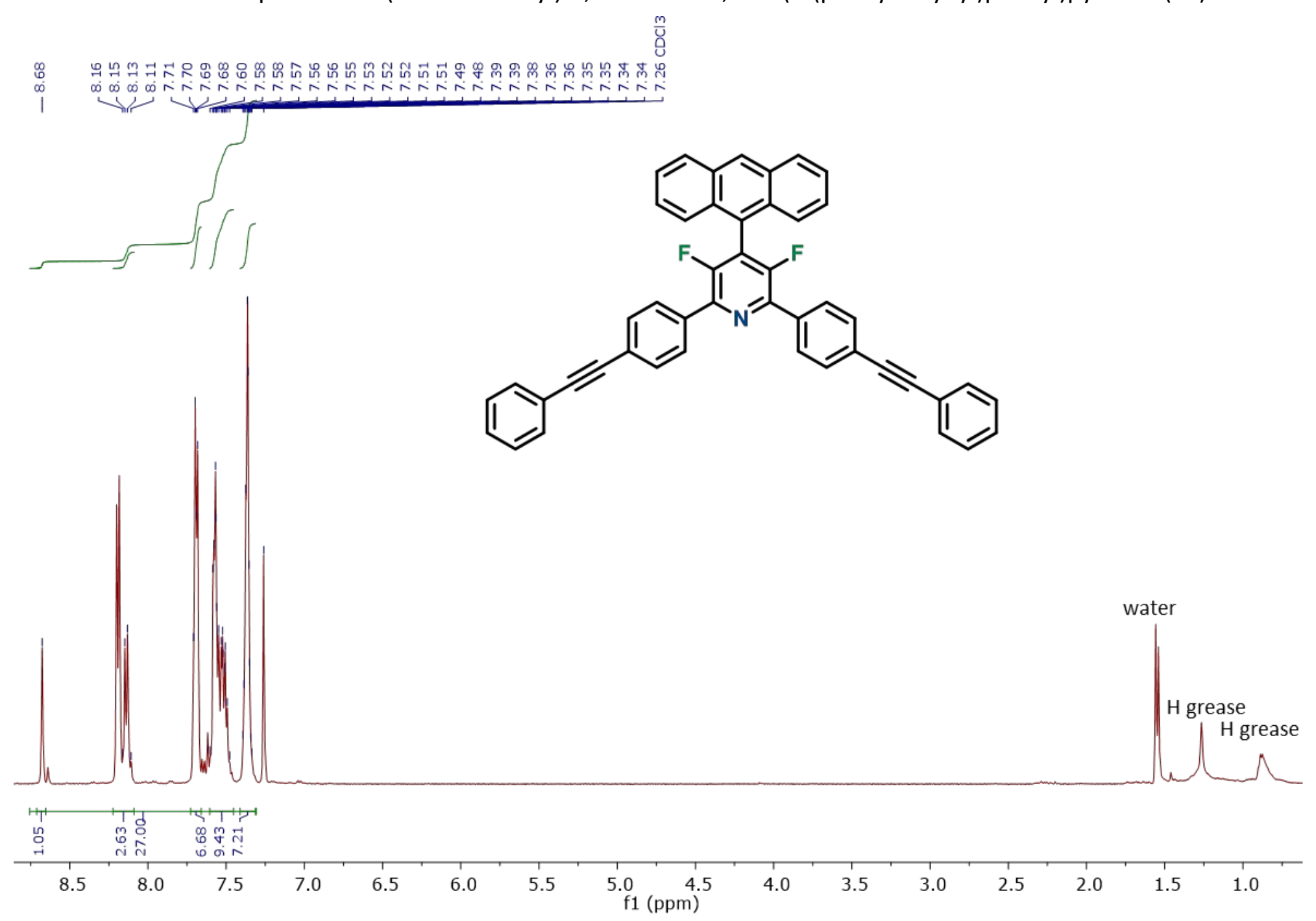


${ }^{19} \mathrm{~F}$ NMR spectra of 2-(anthracen-9-yl)-3,5-difluoro-4,6-bis(4-(phenylethynyl)phenyl)pyridine (2a)

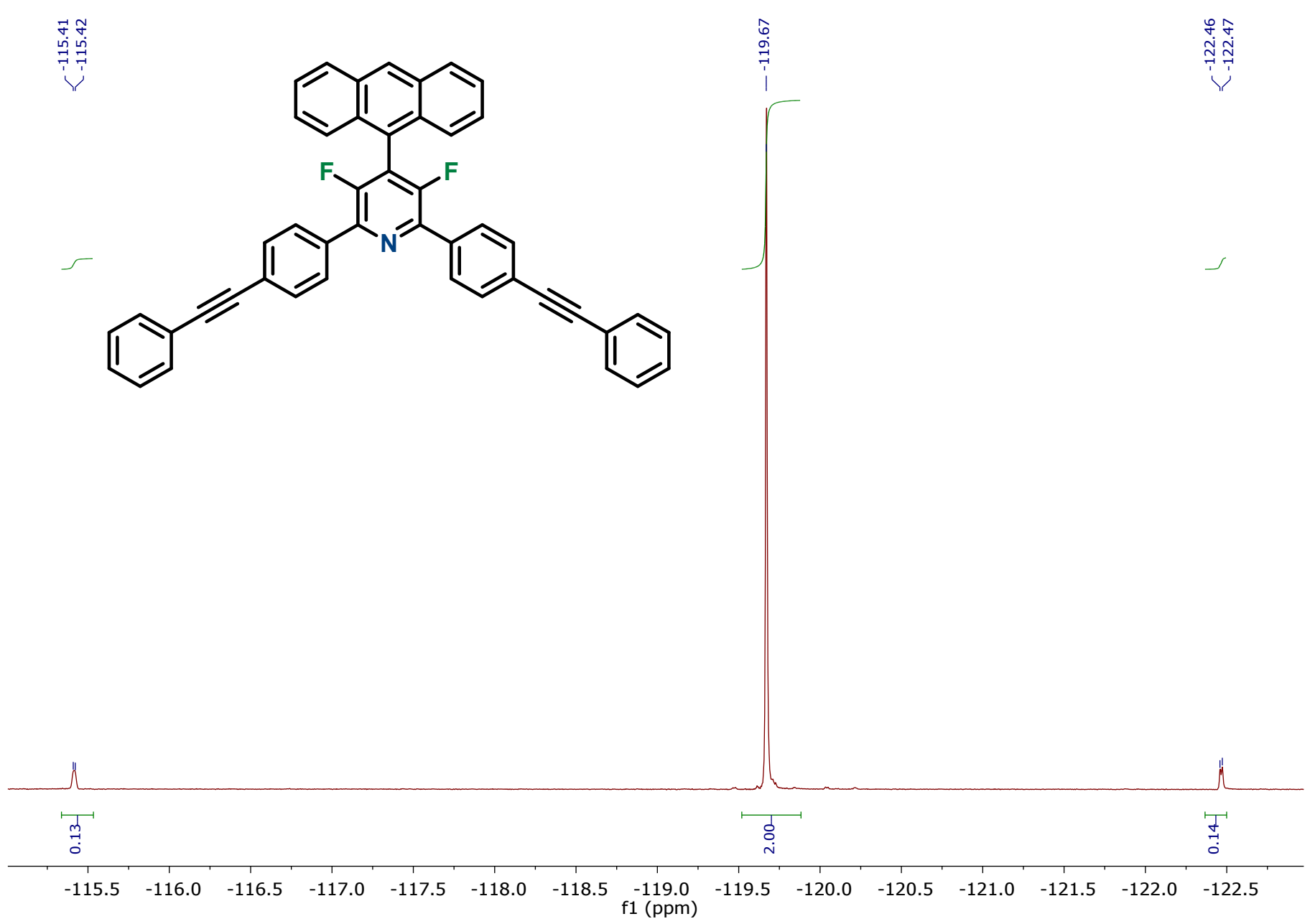


${ }^{13} \mathrm{C}$ NMR spectra of 2-(anthracen-9-yl)-3,5-difluoro-4,6-bis(4-(phenylethynyl)phenyl)pyridine (2a)
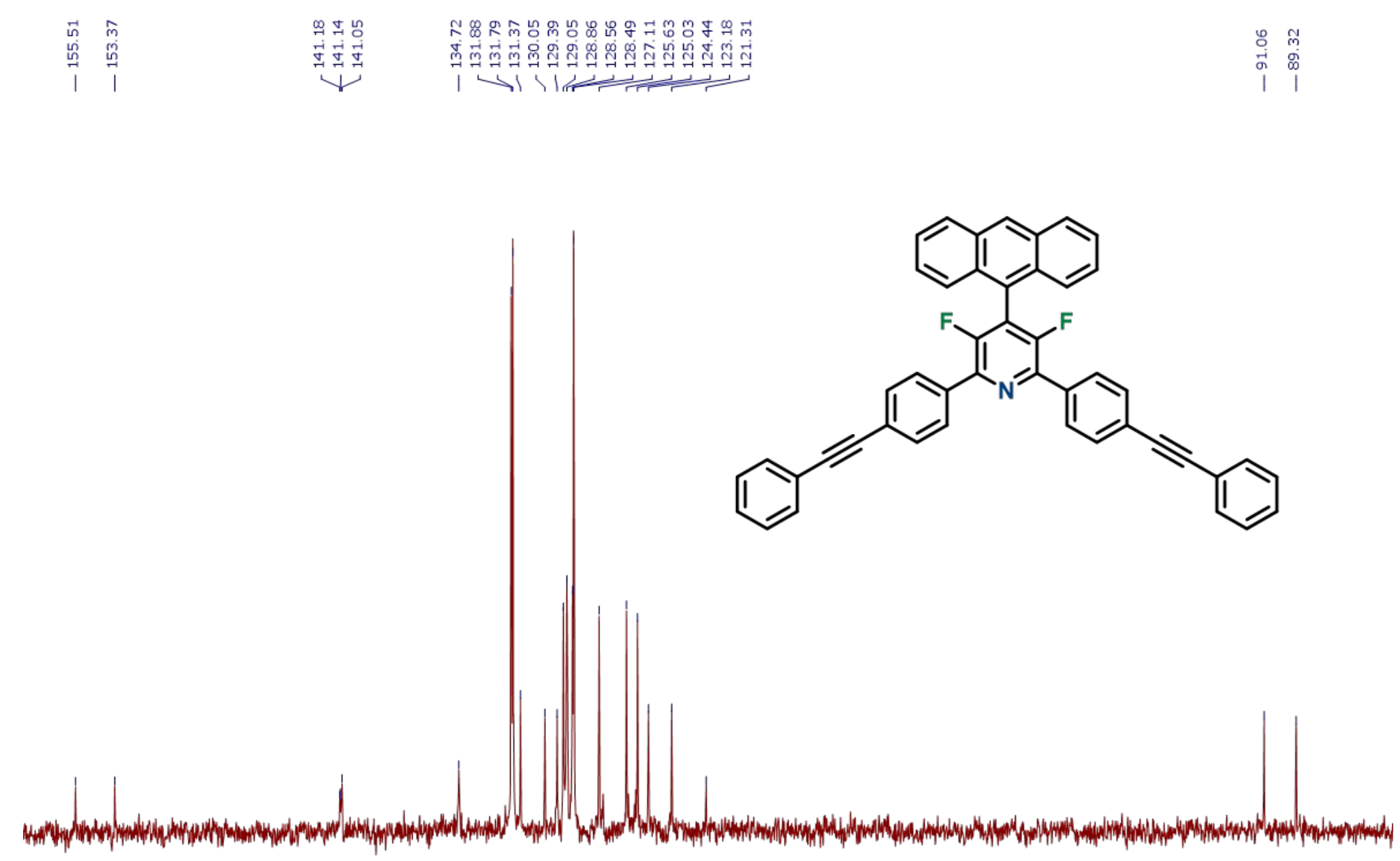
${ }^{1} \mathrm{H}$ NMR spectra of 2,4,6-tri(anthracen-9-yl)-3,5-difluoropyridine (2b)

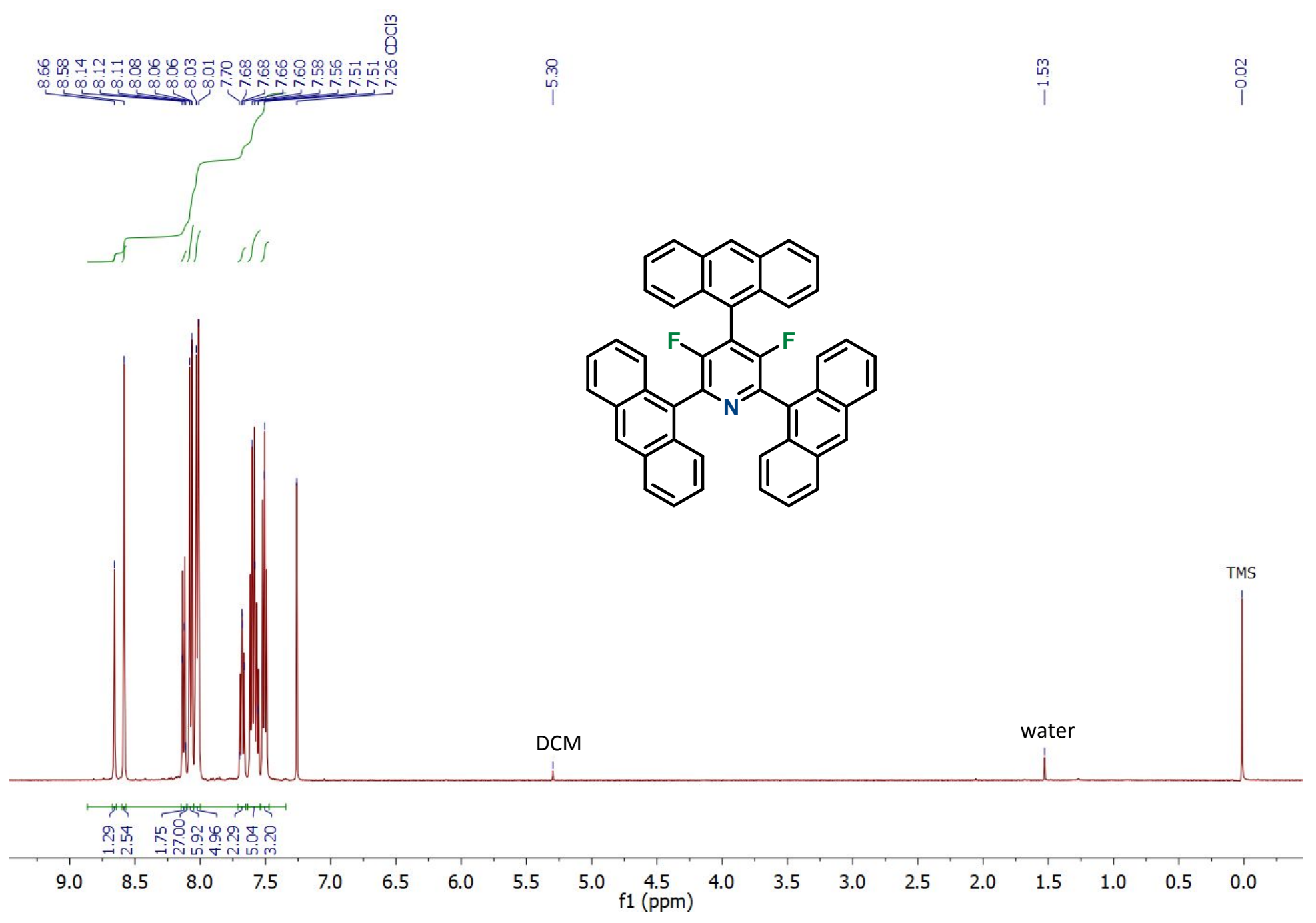


${ }^{19} \mathrm{~F}$ NMR spectra of 2,4,6-tri(anthracen-9-yl)-3,5-difluoropyridine (2b)

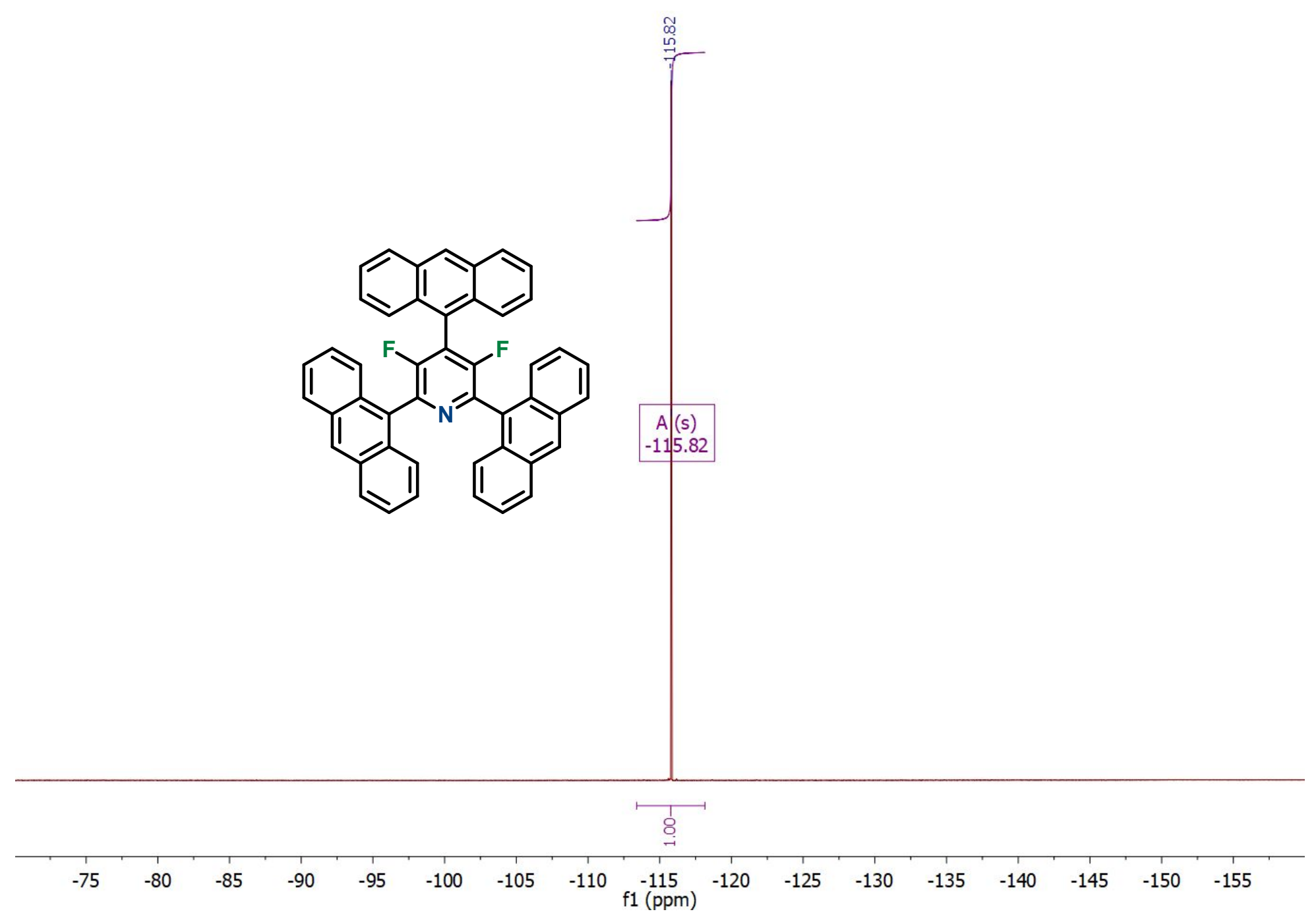


${ }^{13} \mathrm{C}$ NMR spectra of 2,4,6-tri(anthracen-9-yl)-3,5-difluoropyridine (2b)
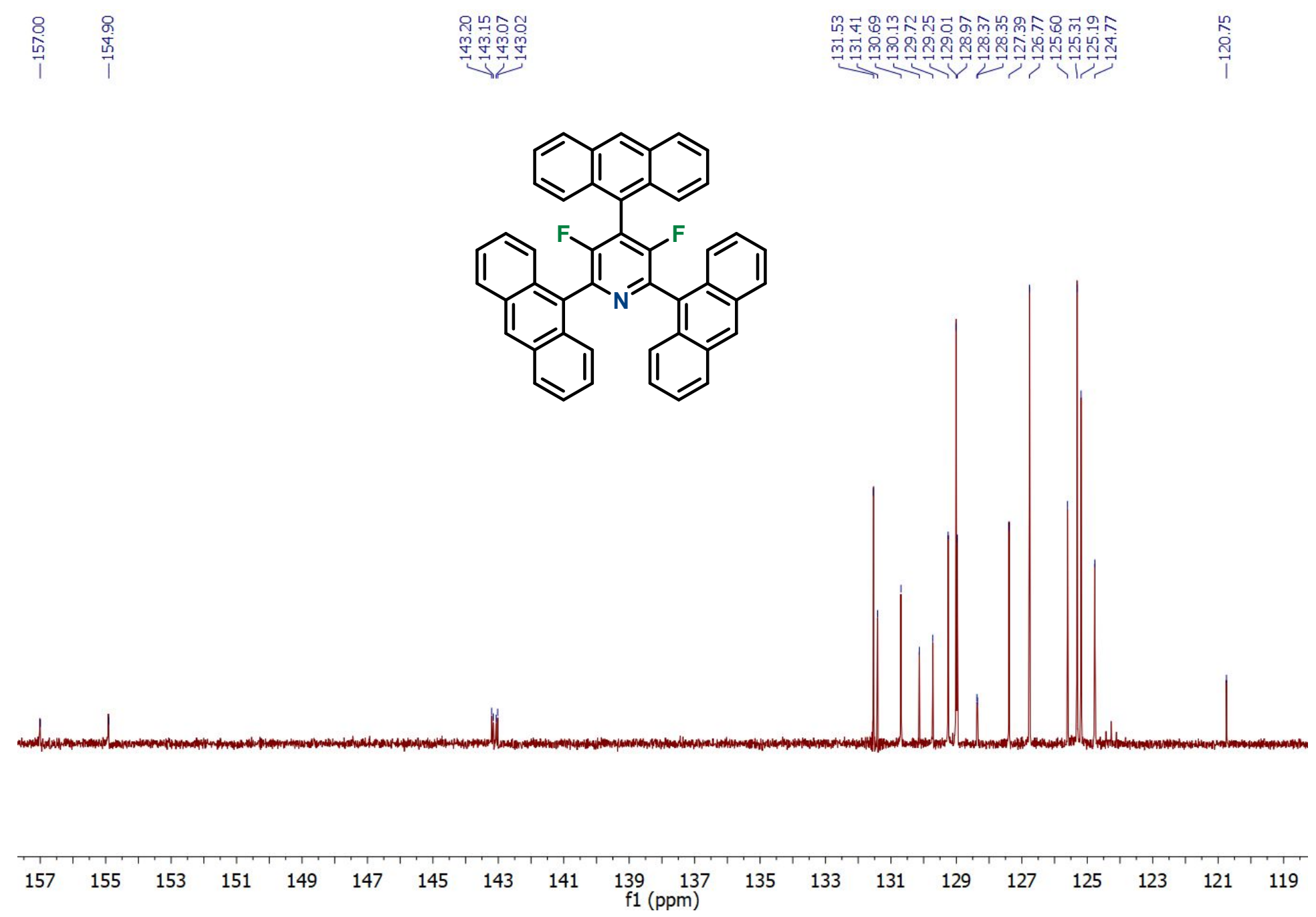


\section{Thermal Analysis}

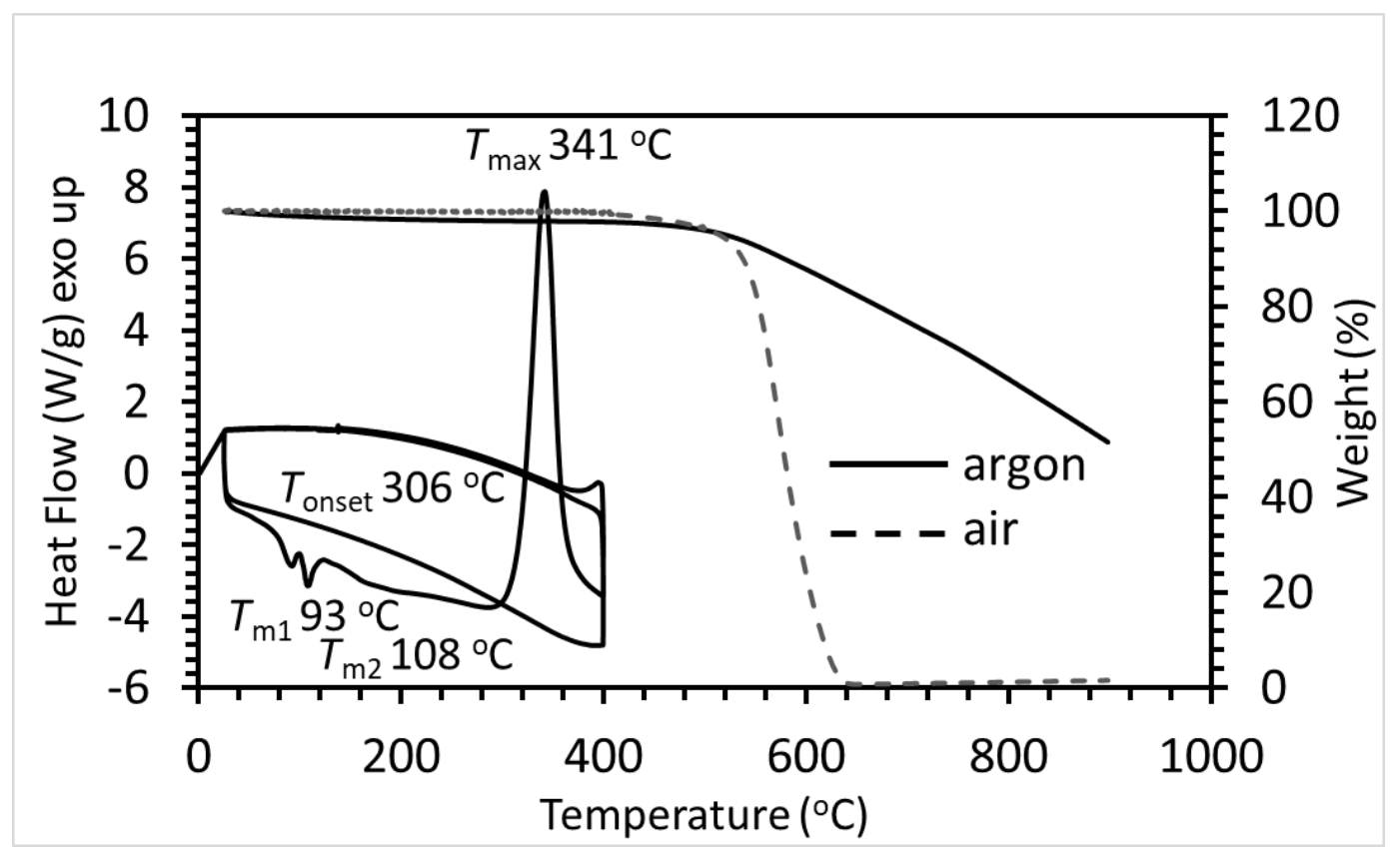

Figure S1. Combined DSC and TGA data of compound (1a). Char yield at $\mathrm{T}_{d}$ in $\operatorname{argon}(96 \%)$. Char Yield at $\mathrm{T}_{d}$ in air (96\%).

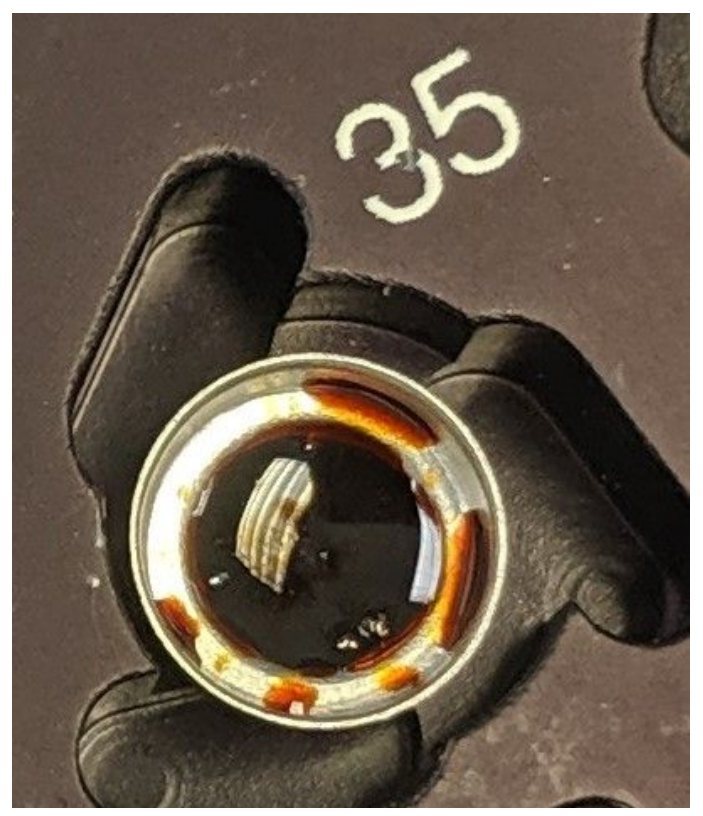

Figure S2. Photo of the network resulting from the curing of 1a. 


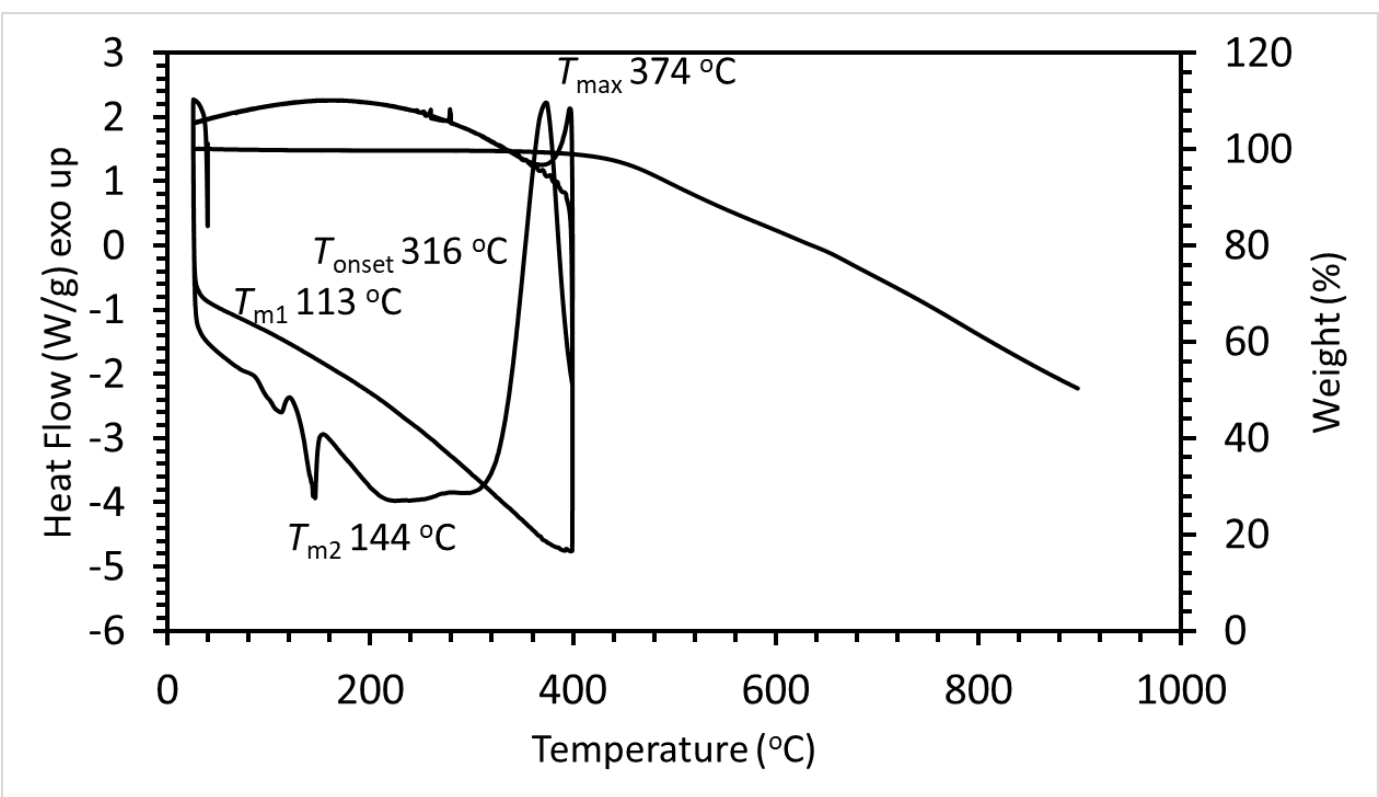

Figure S3. Combined DSC and TGA data of the blended mixture of compounds (1a) and (2b). Char yield at $T_{d}$ in argon (98\%). Char yield at $T_{d}$ in air (95\%).

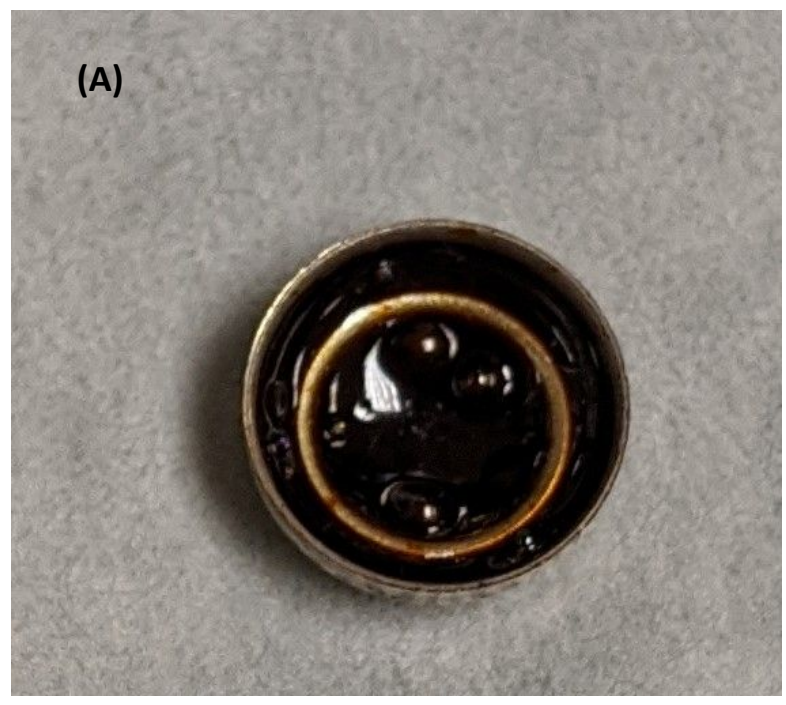

(B)

Figure S4. (A) Photo of the network resulting from the curing of 2a. (B) Photo of the 2a network after being exposed to hot thf and hot dmso for 5 minutes. No change or dissolution was observed. 


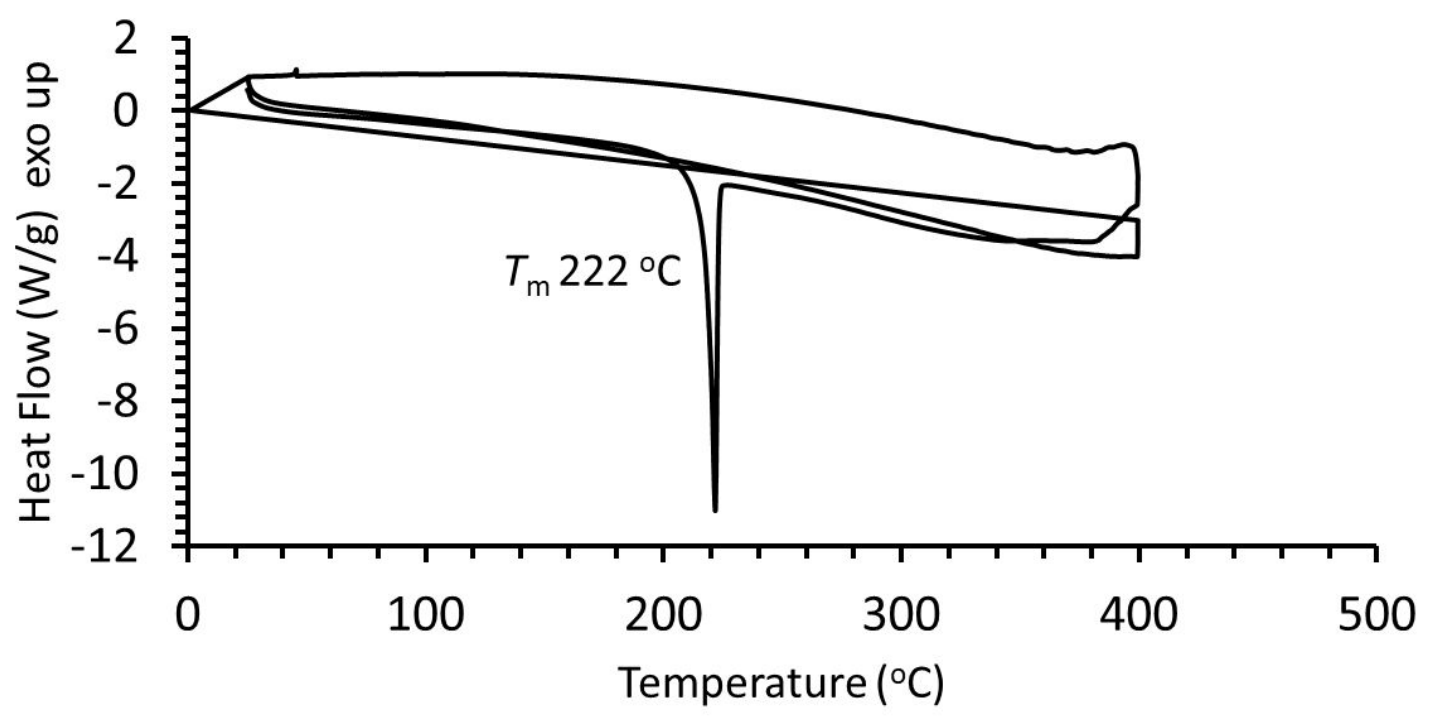

Figure S5. DSC thermogram of compound (1b). 


\section{X-Ray Data}

\section{Computing details}

Data collection: CrysAlis PRO 1.171.40.58a; cell refinement: CrysAlis PRO 1.171.40.58a; data reduction: CrysAlis PRO 1.171.40.58a program(s) used to solve structure. ${ }^{2}$ ShelXT program(s) used to refine structure: SHELXL. ${ }^{3}$ Molecular graphics: Olex2 software used to prepare material for publication: Olex $2 .^{4}$

\section{Experimental details}

\begin{tabular}{|c|c|}
\hline \multicolumn{2}{|l|}{ Crystal data } \\
\hline Chemical formula & $\mathrm{C}_{39} \mathrm{H}_{23} \mathrm{~F}_{2} \mathrm{NO}_{2}$ \\
\hline$M_{\mathrm{r}}$ & 575.58 \\
\hline Crystal system, space group & Triclinic, $P^{-} 1$ \\
\hline Temperature (K) & 100 \\
\hline$a, b, c(\AA)$ & $9.5575(1), 11.9989(1), 13.2803(2)$ \\
\hline$a, b, g\left({ }^{\circ}\right)$ & $102.367(1), 108.958(1), 96.895$ (1) \\
\hline$V\left(\AA^{3}\right)$ & $1376.98(3)$ \\
\hline$z$ & 2 \\
\hline Radiation type & Mo Ka \\
\hline $\mathrm{m}\left(\mathrm{mm}^{-1}\right)$ & 0.09 \\
\hline Crystal size $(\mathrm{mm})$ & $0.26 \times 0.17 \times 0.14$ \\
\hline \multicolumn{2}{|l|}{ Data collection } \\
\hline Diffractometer & $\begin{array}{l}\text { XtaLAB Synergy, Single source at offset/far, } \\
\text { HyPix3000 }\end{array}$ \\
\hline Absorption correction & $\begin{array}{l}\text { Multi-scan } \\
\text { CrysAlis PRO 1.171.40.58a (Rigaku Oxford } \\
\text { Diffraction, 2019) Empirical absorption } \\
\text { correction using spherical harmonics, } \\
\text { implemented in SCALE3 ABSPACK scaling } \\
\text { algorithm. }\end{array}$ \\
\hline$T_{\min }, T_{\max }$ & $0.823,1.000$ \\
\hline $\begin{array}{l}\text { No. of measured, independent and } \\
\text { observed }[I>2 \mathrm{~s}(I)] \text { reflections }\end{array}$ & $33829,5918,4641$ \\
\hline$R_{\text {int }}$ & 0.029 \\
\hline$(\sin q / I)_{\max }\left(\AA^{-1}\right)$ & 0.647 \\
\hline
\end{tabular}




\section{Electronic Supplementary Material (ESI) for Macro Letters.}

This journal is (C) The American Chemical Society 2020

\begin{tabular}{|l|l|}
\hline Refinement \\
\hline$R\left[F^{2}>2 \mathrm{~s}\left(F^{2}\right)\right], w R\left(F^{2}\right), S$ & $0.043,0.117,1.06$ \\
\hline No. of reflections & 5918 \\
\hline No. of parameters & 397 \\
\hline H-atom treatment & H-atom parameters constrained \\
\hline$D \rho_{\max }, \mathrm{D} \rho_{\min }\left(\mathrm{e} \AA^{-3}\right)$ & $0.24,-0.22$ \\
\hline
\end{tabular}

\section{Crystal data}

\begin{tabular}{|l|l|}
\hline $\mathrm{C}_{39} \mathrm{H}_{23} \mathrm{~F}_{2} \mathrm{NO}_{2}$ & $Z=2$ \\
\hline$M_{r}=575.58$ & $F(000)=596$ \\
\hline Triclinic, $P^{-} 1$ & $D_{\mathrm{x}}=1.388 \mathrm{Mg} \mathrm{m}^{-3}$ \\
\hline$a=9.5575(1) \AA$ & Mo Ka radiation, $\mathrm{I}=0.71073 \AA$ \\
\hline$b=11.9989(1) \AA$ & Cell parameters from 17704 reflections \\
\hline$c=13.2803(2) \AA$ & $\mathrm{q}=1.8-27.2^{\circ}$ \\
\hline $\mathrm{a}=102.367(1)^{\circ}$ & $\mathrm{m}=0.09 \mathrm{~mm}^{-1}$ \\
\hline $\mathrm{b}=108.958(1)^{\circ}$ & $T=100 \mathrm{~K}$ \\
\hline $\mathrm{g}=96.895(1)^{\circ}$ & Block, yellow \\
\hline$V=1376.98(3) \AA^{3}$ & $0.26 \times 0.17 \times 0.14 \mathrm{~mm}$ \\
\hline
\end{tabular}

\section{Data collection}

\begin{tabular}{|l|l|}
\hline $\begin{array}{l}\text { XtaLAB Synergy, Single source at offset/far, } \\
\text { HyPix3000 } \\
\text { diffractometer }\end{array}$ & 5918 independent reflections \\
\hline $\begin{array}{l}\text { Radiation source: micro-focus sealed X-ray } \\
\text { tube, PhotonJet (Mo) X-ray Source }\end{array}$ & 4641 reflections with $I>2 \mathrm{~s}(I)$ \\
\hline Mirror monochromator & $R_{\text {int }}=0.029$ \\
\hline Detector resolution: 10.0000 pixels $\mathrm{mm}^{-1}$ & $\mathrm{q}_{\max }=27.4^{\circ}, \mathrm{q}_{\min }=1.7^{\circ}$ \\
\hline w scans & $h=-12^{\circledR} 12$ \\
\hline $\begin{array}{l}\text { Absorption correction: } \text { multi-scan } \\
\text { CrysAlis PRO 1.171.40.58a (Rigaku Oxford } \\
\text { Diffraction, } 2019) \text { Empirical absorption } \\
\text { correction using spherical harmonics, } \\
\text { implemented in SCALE3 ABSPACK scaling } \\
\text { algorithm. }\end{array}$ & $k=-15^{\oplus} 15$ \\
\hline$T_{\min }=0.823, T_{\max }=1.000$ & $I=-16^{\oplus} 16$ \\
\hline 33829 measured reflections & \\
\hline
\end{tabular}




\section{Refinement}

\begin{tabular}{|l|l|}
\hline Refinement on $F^{2}$ & 0 restraints \\
\hline Least-squares matrix: full & $\begin{array}{l}\text { Hydrogen site location: inferred from } \\
\text { neighbouring sites }\end{array}$ \\
\hline$R\left[F^{2}>2 \mathrm{~s}\left(F^{2}\right)\right]=0.043$ & $\mathrm{H}$-atom parameters constrained \\
\hline$W R\left(F^{2}\right)=0.117$ & $\begin{array}{l}W=1 /\left[\mathrm{s}^{2}\left(F_{\mathrm{o}}^{2}\right)+(0.0535 P)^{2}+0.3614 P\right] \\
\text { where } P=\left(F_{\mathrm{o}}^{2}+2 F_{\mathrm{c}}^{2}\right) / 3\end{array}$ \\
\hline$S=1.06$ & $(\mathrm{D} / \mathrm{s})_{\max }<0.001$ \\
\hline 5918 reflections & $\mathrm{D} \rho_{\max }=0.24$ e $\AA^{-3}$ \\
\hline 397 parameters & $\mathrm{D} \rho_{\min }=-0.22$ e $\AA^{-3}$ \\
\hline
\end{tabular}

\section{Special details}

Geometry. All esds (except the esd in the dihedral angle between two I.s. planes) are estimated using the full covariance matrix. The cell esds are taken into account individually in the estimation of esds in distances, angles and torsion angles; correlations between esds in cell parameters are only used when they are defined by crystal symmetry. An approximate (isotropic) treatment of cell esds is used for estimating esds involving l.s. planes.

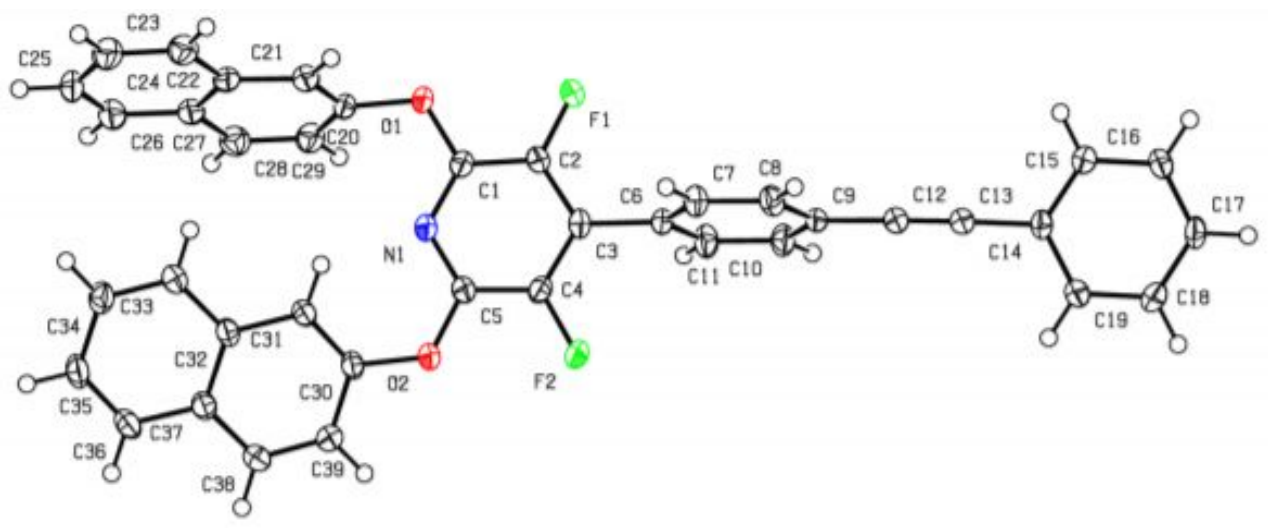

Figure S6. ORTEP Diagram of 3,5-difluoro-2,6-bis(naphthalen-1-yloxy)-4-(4(phenylethynyl)phenyl)pyridine (1b). 
Table S1. Selected bond angles of 3,5-difluoro-2,6-bis(naphthalen-1-yloxy)-4-(4(phenylethynyl)phenyl)pyridine (1b).

\begin{tabular}{|c|c|c|c|c|c|c|c|}
\hline \multicolumn{1}{|c|}{ Ring Bond Angles ( $\left.{ }^{\circ}\right)$} \\
\hline N1-C1-C2 & C1-C2-C3 & C2-C3-C4 & C3-C4-C5 & C4-C5-N1 & C2-C3-C6 & C1-C2-F1 & C5-C4-F2 \\
\hline $122.30(12)$ & $120.02(12)$ & $116.25(12)$ & $120.60(12)$ & $121.45(12)$ & $122.02(12)$ & $120.25(12)$ & $119.68(12)$ \\
\hline
\end{tabular}

Table S2. Selected bond lengths of 3,5-difluoro-2,6-bis(naphthalen-1-yloxy)-4-(4(phenylethynyl)phenyl)pyridine (1b).

\begin{tabular}{|c|c|c|c|c|c|c|c|}
\hline \multicolumn{5}{|c|}{ Ring Bond Distances (Å) } & \multicolumn{3}{c|}{ (Å) } \\
\hline N1-C1 & C1-C2 & C2-C3 & C3-C4 & C4-C5 & C2-F1 & C4-F2 & C3-C6 \\
\hline $1.3177(17)$ & $1.3824(19)$ & $1.3871(19)$ & $1.3840(19)$ & $1.3873(18)$ & $1.3512(15)$ & $1.3503(15)$ & $1.4936(18)$ \\
\hline
\end{tabular}

\section{References}

1. Fulmer, G. R.; Miller, A. J.; Sherden, N. H.; Gottlieb, H. E.; Nudelman, A.; Stoltz, B. M.; Bercaw, J. E.; Goldberg, K. I. Organometallics. 2010, 29, 2176-2179

2. Rigaku, OD. CrysAlis PRO. Rigaku Oxford Diffraction Ltd, Yarnton, England, 2018.

3. Sheldrick, G. M. Acta Cryst. 2015, 71, 3-8.

4. Dolomanov, O. V.; Bourhis, L. J.; Gildea, R. J.; Howard, J. A. K; Puschmann, H. J. Appl. Cryst. 2009, 42, 339-341 